\title{
CHARACTERISTIC LAPLACIAN IN SUB-RIEMANNIAN GEOMETRY
}

\author{
JEREMY DANIEL AND XIAONAN MA
}

\begin{abstract}
We study a Laplacian operator related to the characteristic cohomology of a smooth manifold endowed with a distribution. We prove that this Laplacian does not behave very well: it is not hypoelliptic in general and does not respect the bigrading on forms in a complex setting. We also discuss the consequences of these negative results for a conjecture of P. Griffiths, concerning the characteristic cohomology of period domains.
\end{abstract}

\section{INTRODUCTION}

Let $X$ be a smooth manifold and denote by $\Omega^{\bullet}(X)$ its differential graded algebra of smooth differential forms. Given a constant-rank distribution $W$ on $X$, we consider the Pfaffian system associated to $W$, that is the graded differential ideal $\mathcal{J}^{\bullet}$ generated by the smooth global sections of the annihilator of $W$ in $T^{*} X$. Pfaffian systems constitute an important class of exterior differential systems, for which we refer the interested reader to [2].

For a differential map $f$ from a smooth manifold $Y$ to $X$, it is equivalent for the differential of $f$ to have its values in the distribution $W$ and for the pullback by $f$ of any form in $\mathcal{J}$ to vanish on $Y$; we call such maps solutions of the Pfaffian system. Hence, it is reasonable to consider the complex $\Omega^{\bullet}(X) / \mathcal{J}^{\bullet}$, endowed with the differential induced by exterior differentiation on $\Omega^{\bullet}(X)$. This is well defined since $\mathcal{J}$ is stable by exterior differentiation. We define the characteristic cohomology of $(X, W)$ to be the cohomology of this complex and we denote it by $H_{\mathcal{J}}(X)$. More generally, we will attach the adjective characteristic to the constructions related to the Pfaffian system. In [3] and [4, this characteristic cohomology is intensively studied. Remark that if $f: Y \rightarrow X$ is a solution of $\mathcal{J}$, then one has a map in cohomology

$$
f^{*}: H_{\mathcal{J}}^{\bullet}(X) \rightarrow H^{\bullet}(Y) .
$$

In [10, $\S I I I]$, the characteristic cohomology of period domains is the object of an interesting conjecture. Period domains $D$ are homogeneous spaces $G / V$, where $V$ is a compact subgroup of a Lie group $G$, encountered in the study of variations of Hodge structures. To every variation of Hodge structures over a complex manifold $S$, one can construct a holomorphic map from $S$ to a quotient $\Gamma \backslash D$, where $\Gamma$ is a discrete subgroup of $G$, and the well-known Griffiths transversality condition (a.k.a infinitesimal period relation, see e.g. [5]) states that the differential of such a map has its values in some distribution $W$ of $\Gamma \backslash D$, coming from a $G$-invariant distribution of $D$. The conjecture can be stated as follows:

Conjecture 0.1. Let $\Gamma$ be a cocompact subgroup of $G$, acting freely on $D=G / V$. If the distribution $W$ is bracket-generating and if $m \leq m_{0}$, where $m_{0}$ is some integer

Date: June 6, 2018. 
determined by the Pfaffian system $\mathcal{J}$ associated to $W$, then $H_{\mathcal{J}}^{m}(\Gamma \backslash D)$ carries a natural pure real Hodge structure of weight $m$.

A real pure Hodge structure of weight $m$ is a real vector space $E$ whose complexification $E \otimes_{\mathbb{R}} \mathbb{C}$ carries a decomposition

$$
E \otimes_{\mathbb{R}} \mathbb{C}=\bigoplus_{p+q=m} E^{p, q}
$$

where $E^{p, q}$ are complex vector spaces satisfying $E^{p, q}=\overline{E^{q, p}}$. The prototype of real pure Hodge structure of weight $m$ is the real cohomology in degree $m$ of a compact Kähler manifold, as results from Hodge theory (especially the ellipticity of the Hodge Laplacian) and Kähler identities (see [9] or [12] for instance). One idea to study this conjecture is to develop an analogue of Hodge theory in this characteristic situation. More precisely, following [8], one defines a Laplacian related to the Pfaffian system and one can try to prove that there is an isomorphism between its harmonic forms and the characteristic cohomology. In the complex setting, if the Laplacian respects the bigrading on forms, we get a Hodge structure on harmonic forms, hence on characteristic cohomology. However, we will see that the picture is not so bright and that Conjecture 0.1 certainly needs to be studied in another way.

In the first part, we construct this characteristic Laplacian. In [8, §III. A], it is asserted that this characteristic Laplacian is hypoelliptic and that in this case we have an isomorphism of the characteristic cohomology with the space of harmonic forms. However, we explain why there seems to be no reason for the hypoellipticity of the characteristic Laplacian in general and, in Example 1.10, we give an explicit counterexample to the hypoellipticity in a complex setting:

Proposition 0.2. One can construct compact complex manifolds of dimension 3, endowed with a contact structure, for which the corresponding characteristic Laplacian is not hypoelliptic in degree 2.

In particular, it seems difficult to understand the characteristic cohomology via harmonic forms.

In the second part, we nevertheless study the characteristic Laplacian in more details and answer the following question, asked in [8, §III. A]:

Question 0.3. Let $(X, h)$ be a hermitian manifold, endowed with a holomorphic distribution $W$. Let $W_{\mathbb{R}}$ denote the underlying real distribution in $T X$ and consider the Pfaffian system associated to $W_{\mathbb{R}}$. What are the necessary and sufficient conditions for the characteristic Laplacian to respect the bigrading on differential forms on $X$ ?

We give an unexpected answer to this question in Theorem 2.1;

Theorem 0.4. The characteristic Laplacian never respects the bigrading when the distribution $W$ is not involutive (in particular, when it is bracket-generating, as in Conjecture $0.1)$.

This is quite deceptive since it shows that there is nothing like a Kähler condition in the characteristic case. Indeed, in the classical case where the distribution $W$ is the whole space $T X$, we show in Theorem 2.2 : 
Theorem 0.5. A complex Hermitian manifold is Kähler if and only if the Hodge Laplacian preserves the bigrading on differential forms on $X$.

It seems that the necessity part was not written yet in the literature. This result and its proof are independant of the rest of the article.

In this paper, if $E$ is a complex vector bundle on a manifold $X$, we will denote by $E_{\mathbb{R}}$ its underlying real vector bundle. Moreover, $\wedge$ and $i$ are the exterior and interior products on $\Omega(X)$ and all distributions are assumed to be of constant rank.

Acknowledgments. We thank Professors R. Bryant and P. Griffiths for useful discussions and for sending us M. Taylor's unpublished work. X. Ma thanks the Institut Universitaire de France for support.

\section{The CHARACTERISTIC LAPLACIAN}

This section is organized as follows. In Subsection 1.1, we define the characteristic Laplacian associated with a distribution for Riemannian manifolds. In Subsection 1.2, we explain Taylor's counterexample for the hypoellipticity of the characteristic Laplacian. In Subsection 1.3, we explain why the hypoellipticity does not seem to hold in general by computing its principal symbol. In Subsection 1.4, we give a counterexample for the hypoellipticity of the characteristic Laplacian in the complex setting which is the context of the original question.

1.1. Definitions and notations. Let $\left(X, g^{T X}\right)$ be a smooth compact Riemannian manifold, endowed with a (constant-rank) distribution $W$. We denote by $F$ the annihilator of $W$ in $T^{*} X$; it is a vector subbundle of $T^{*} X$. We denote by $\Omega^{\bullet}(X)$ the graded algebra of differential forms on $X$ and we endow it with the natural metric $g^{\Omega(X)}$ induced by $g^{T X}$. We consider

- $\mathcal{I}$ the algebraic ideal generated by the smooth sections $I_{X}=\mathscr{C}^{\infty}(X, F)$;

- $\mathcal{J}$ the differential ideal generated by the smooth sections of $F$ on $X$, that is the minimal algebraic ideal containing the smooth sections of $F$ and stable by exterior differentiation.

Remark that $\mathcal{J}$ is the algebraic ideal generated by $I_{X}$ and $d I_{X}$. If $\left(\theta_{j}\right)$ is a frame of $F$, the forms in $\mathcal{I}$ can locally be written as

$$
\sum_{j} \theta_{j} \wedge \phi_{j}
$$

where $\phi_{j}$ are arbitrary forms on $X$, and those in $\mathcal{J}$ are of the form

$$
\sum_{j} \theta_{j} \wedge \phi_{j}+d \theta_{j} \wedge \psi_{j}
$$

where $\phi_{j}, \psi_{j}$ are arbitrary forms on $X$.

Let $\mathcal{Q}$ be the orthogonal complement of $\mathcal{J}$ in $\Omega(X)$. Remark that $\mathcal{Q}$ is naturally graded. We define a differential operator for forms in $\mathcal{Q}$ by

$$
d_{\mathcal{Q}}:=\pi_{\mathcal{Q}} \circ d \circ \pi_{\mathcal{Q}}: \mathcal{Q} \rightarrow \mathcal{Q},
$$


where $\pi_{\mathcal{Q}}$ is the orthogonal projection from $\Omega(X)$ onto $\mathcal{Q}$. Since $\mathcal{J}$ is stable by $d$, we have

$$
\pi_{\mathcal{Q}} \circ d \circ \pi_{\mathcal{Q}}=\pi_{\mathcal{Q}} \circ d: \Omega^{\bullet}(X) \rightarrow \Omega^{\bullet}(X)
$$

By (1.2), we know

$$
d_{\mathcal{Q}}^{2}=\pi_{\mathcal{Q}} \circ d \circ \pi_{\mathcal{Q}} \circ d \circ \pi_{\mathcal{Q}}=\pi_{\mathcal{Q}} \circ d^{2} \circ \pi_{\mathcal{Q}}=0 .
$$

Since $\mathcal{Q}$ is the orthogonal complement of $\mathcal{J}$ and $\mathcal{J}$ is stable by $d$, we know that $\mathcal{Q}$ is stable by the adjoint $d^{*}$ of $d$ and the restriction of $d^{*}$ to $\mathcal{Q}$ is $d_{\mathcal{Q}}^{*}$, the adjoint of $d_{\mathcal{Q}}$ for the natural $L^{2}$-structure on $\mathcal{Q}$. Indeed, if $\alpha$ (resp. $\beta$ ) is in $\mathcal{Q}$ (resp. $\mathcal{J}$ ), then $d \beta$ is in $\mathcal{J}$ and this implies

$$
\left(d^{*} \alpha, \beta\right)=(\alpha, d \beta)=0 .
$$

Since this is true for any $\beta$ in $\mathcal{J}, d^{*} \alpha$ is in $\mathcal{Q}$. Moreover, if $\alpha$ and $\beta$ are in $\mathcal{Q}$, then

$$
\left(d^{*} \alpha, \beta\right)=(\alpha, d \beta)=\left(\alpha, d_{\mathcal{Q}} \beta\right)
$$

and this shows that $d_{\mathcal{Q}}^{*} \alpha=d^{*} \alpha$.

Definition 1.1. The characteristic Laplacian $\Delta_{\mathcal{Q}}$ on $X$ with respect to $W$ is the differential operator on $\mathcal{Q}$

$$
\Delta_{\mathcal{Q}}=d_{\mathcal{Q}} d_{\mathcal{Q}}^{*}+d_{\mathcal{Q}}^{*} d_{\mathcal{Q}}: \mathcal{Q} \rightarrow \mathcal{Q}
$$

Remark 1.2. In sub-Riemmanian geometry (where the distribution $W$ is involutive), one defines a sub-Laplacian on functions (see [13]). This sub-Laplacian is hypoelliptic and coincides with the characteristic Laplacian in degree 0. In Example 1.5, we will see that hypoellipticity can fail in positive degrees.

Remember that we defined the characteristic cohomology of $X$ (associated to the distribution $W$ ) to be

$$
H_{\mathcal{J}}^{\bullet}(X):=H^{\bullet}\left(\Omega^{\bullet}(X) / \mathcal{J}^{\bullet}, d\right),
$$

with the differential induced by exterior differentiation on $\Omega^{\bullet}(X)$.

By definition of $\mathcal{Q}$ and $d_{Q}$, this characteristic cohomology is naturally isomorphic to the cohomology of the complex $\left(\mathcal{Q}^{\bullet}, d_{Q}\right)$. An analogue of Hodge theory would be the following conjecture.

Conjecture 1.3. We denote by $\mathcal{H}_{\mathcal{Q}}^{\bullet}(X)$ the characteristic harmonic forms, that is the kernel of $\Delta_{\mathcal{Q}}$. Then

- $\mathcal{H}_{\mathcal{Q}}^{\bullet}(X)$ is of finite dimension.

- There is an orthogonal decomposition

$$
\mathcal{Q}^{\bullet}(X)=\mathcal{H}_{\mathcal{Q}}^{\bullet}(X) \oplus d_{\mathcal{Q}}\left(\mathcal{Q}^{\bullet-1}(X)\right) \oplus d_{\mathcal{Q}}^{*}\left(\mathcal{Q}^{\bullet+1}(X)\right) .
$$

- The natural application $\mathcal{H}_{\mathcal{Q}}^{\bullet}(X) \rightarrow H_{\mathcal{J}}^{\bullet}(X)$ is an isomorphism.

In classical Hodge theory, one gets these results as consequences of the ellipticity of the Laplacian. In the next section, we show that the characteristic Laplacian in not even hypoelliptic in general. Hence we cannot really hope this conjecture to be true. 
1.2. The question of hypoellipticity. We recall that, if $E$ and $F$ are vector bundles over $X$ and $P: E \rightarrow F$ is a differential operator, then $P$ is said to be hypoelliptic if the following condition is satisfied: for every local distribution $u$ with values in $E$, if $P u$ is smooth on an open set $U \subset X$, then the restriction of $u$ to $U$ is smooth. Elliptic operators, like the usual Hodge Laplacian, are hypoelliptic. It is a natural question to ask whether $\Delta_{\mathcal{Q}}$ is hypoelliptic since this would be the first step in order to prove Conjecture 1.3 .

The most known sufficient condition for a second order differential operator to be hypoelliptic is due to Hörmander ([1] $)$.

Theorem 1.4 (Sum of squares condition of hypoellipticity). Let $P$ be a second order differential operator from a vector bundle $E$ to itself. Suppose that locally one can find smooth vector fields $X_{0}, \ldots, X_{k}$ and a smooth function $c$ such that in a local frame of $E$,

$$
P u=\left(\sum_{i=1}^{k} X_{i}^{2}+X_{0}+c\right) u
$$

(in particular $P$ acts componentwise). Then $P$ is hypoelliptic if and only if $X_{0}, \ldots, X_{k}$ generate $T X$ by brackets.

In [8], it is suggested that this theorem implies that the characteristic Laplacian is hypoelliptic when the distribution $W$ is bracket-generating. We will first give a counterexample due to Michael Taylor [14] and then compute the principal symbol of $\Delta_{\mathcal{Q}}$ in order to understand why the hypoellipticity certainly fails in general.

Example 1.5. A contact structure on a 3-manifold $M$ is one of the simplest examples of Pfaffian systems. It is the datum of a 2-rank distribution $W$ on $M$ which is bracketgenerating. For instance, one can take for $W$ the kernel of the 1 -form $\theta=d u-p d x$ in coordinates $(x, u, p)$ in $M=\mathbb{R}^{3}$. A natural example in which $M$ is compact is constructed as follows: we consider $\mathcal{H}^{3}$ the 3 -dimension Heisenberg group, that is $\mathbb{R}^{3}$ with coordinates $(p, q, t)$ and group structure $(p, q, t) \cdot\left(p^{\prime}, q^{\prime}, t^{\prime}\right)=\left(p+p^{\prime}, q+q^{\prime}, t+t^{\prime}+\frac{1}{2}\left(p q^{\prime}-p^{\prime} q\right)\right)$. One checks that the 1 -form $\theta=d t-\frac{1}{2} q d p+\frac{1}{2} p d q$ is right-invariant and defines a contact structure on $\mathcal{H}^{3}$. Taking a cocompact discrete subgroup $\Gamma$ of $G, M=G / \Gamma$ still carries the contact structure.

Locally, all contact structures on 3-manifolds are the same. Let $M$ be a 3-dimensional Riemannian manifold with a contact form $\theta$ and corresponding 2-rank distribution $W$. Let $U$ be an open set in $M$. Then

- $\mathcal{J}^{0}(U)=0$

- $\mathcal{J}^{1}(U)=\mathscr{C}^{\infty}(U, \mathbb{R} \theta)$,

- $\mathcal{J}^{2}(U)=\Omega^{2}(U), \mathcal{J}^{3}(U)=\Omega^{3}(U)$,

and

- $\mathcal{Q}^{0}(U)=\mathscr{C}^{\infty}(U)$,

- $\mathcal{Q}^{1}(U)=\mathscr{C}^{\infty}(U, E)$,

- $\mathcal{Q}^{2}(U)=0, \mathcal{Q}^{3}(U)=0$,

where $E \rightarrow U$ is the real vector bundle of rank 2 , which is the orthogonal of $\mathbb{R} \theta$ in $T^{*} \mathbb{R}^{3}$. In degree 1, the characteristic Laplacian is well-defined on forms with compact support. We have

$$
\Delta_{\mathcal{Q}}^{1}=d_{\mathcal{Q}} d_{\mathcal{Q}}^{*}: \mathscr{C}_{c}^{\infty}(U, E) \rightarrow \mathscr{C}_{c}^{\infty}(U, E)
$$


and one can consider it as a second order differential operator on $E$. Let us denote by $\left(X_{1}, X_{2}\right)$ a (smooth) orthonormal frame of $W$ over $\bar{U}$ and by $\left(\alpha^{1}, \alpha^{2}\right)$ the dual frame of $W^{*}$. Using the metric, $W^{*}$ can be seen as a subbundle of $T^{*} M$ and $E$ can be identified with $W^{*}$ (see also the following subsection [1.3).

A form $\mu \in L^{2}(U, E)$ can thus be written $\mu=\mu_{1} \alpha^{1}+\mu_{2} \alpha^{2}$, with $\mu_{i} \in L^{2}(U, \mathbb{R})$, and $d_{\mathcal{Q}}^{*} \mu$ is given by

$$
d_{\mathcal{Q}}^{*} \mu=Y_{1} \mu_{1}+Y_{2} \mu_{2},
$$

where $Y_{1}, Y_{2}$ are first order scalar differential operators on $U$.

In particular

$$
d_{\mathcal{Q}}^{*}\left(\mu_{1} \alpha^{1}\right)=Y_{1} \mu_{1}
$$

We claim that $Y_{1} \mu_{1}$ can be smooth (even zero) without $\mu_{1}$ being smooth. Indeed, in some local system of centered coordinates $\left(x_{1}, x_{2}, x_{3}\right)$, star-shaped in $0, Y_{1}$ has the form

$$
Y_{1}=\frac{\partial}{\partial x_{1}}+f\left(x_{1}, x_{2}, x_{3}\right)
$$

where $f$ is some smooth function. Consider the function

$$
v\left(x_{1}, x_{2}, x_{3}\right)=\exp \left(\int_{0}^{x_{1}} f\left(t, x_{2}, x_{3}\right) d t\right) \mu_{1}\left(x_{1}, x_{2}, x_{3}\right)
$$

which is smooth if and only if $\mu_{1}$ is smooth. We compute that

$$
\frac{\partial}{\partial x_{1}} v\left(x_{1}, x_{2}, x_{3}\right)=\exp \left(\int_{0}^{x_{1}} f\left(t, x_{2}, x_{3}\right) d t\right) Y_{1} \mu_{1}\left(x_{1}, x_{2}, x_{3}\right) \text {. }
$$

Choosing $v$ independant of $x_{1}$ but not smooth, one has $Y_{1} \mu_{1}=0$, proving the claim.

Remark 1.6. In an analogous example occurring in the complex situation (Example 1.10), we will need to be more precise. In particular, formula (1.8) can be made explicit:

$$
d_{\mathcal{Q}}^{*} \mu=-\operatorname{div}\left(\mu_{1} X_{1}+\mu_{2} X_{2}\right),
$$

where $\operatorname{div}(X)$ is the divergence of the vector field $X$.

In order to better understand why the hypoellipticity seems to fail, we will compute the principal symbol of $\Delta_{\mathcal{Q}}$. It will not show that $\Delta_{\mathcal{Q}}$ is not hypoelliptic but it will at least show that the sum of squares condition cannot be applied, at least if one only considers the second order terms.

1.3. The principal symbol of the characteristic Laplacian. Recall that we denote by $F$ the annihilator of $W$ in $T^{*} X$. Let $N$ be the orthogonal complement of $W$ in $\left(T X, g^{T X}\right)$. Then as a smooth vector bundle, we have

$$
T X=W \oplus N, \quad \text { and } \quad T^{*} X=W^{*} \oplus N^{*} .
$$

We can identify $N^{*}$ and $F$ as $\mathscr{C}^{\infty}$ vector bundles, and

$$
\mathcal{I}=\mathscr{C}^{\infty}\left(X, N^{*} \widehat{\otimes} \Lambda\left(T^{*} X\right)\right) .
$$

The quotient $\Omega(X) / \mathcal{I}$ can be identified with the orthogonal complement of $\mathcal{I}$, i.e.,

$$
\Omega_{W}(X):=\mathscr{C}^{\infty}\left(X, \Lambda W^{*}\right) .
$$

In what follows, we do these identifications without further notice. 
Since $\mathcal{I} \subset \mathcal{J}, \mathcal{Q}=\mathcal{J}^{\perp}$ can be viewed as a subspace of $\Omega_{W}(X)$. The orthogonal complement of $\mathcal{Q}$ in $\Omega_{W}(X)$ will be identified with $\mathcal{J} / \mathcal{I}$. We thus have the following decompositions:

$$
\Omega(X)=\mathcal{I} \oplus \Omega_{W}(X)
$$

and

$$
\Omega_{W}(X)=\mathcal{J} / \mathcal{I} \oplus \mathcal{Q}
$$

All of these spaces are naturally graded. We define a map $\varphi: F \rightarrow \Lambda^{2}\left(W^{*}\right)$ by: for $\theta \in \mathscr{C}^{\infty}(X, F), v, w \in \mathscr{C}^{\infty}(X, W)$, set

$$
\varphi(\theta)(v, w):=(d \theta)(v, w)=-\theta([v, w]) .
$$

We check that for any $x \in X, \varphi(\theta)(v, w)_{x}$ depends only on $\theta_{x}, v_{x}$ and $w_{x}$.

The map $\varphi: F \rightarrow \Lambda^{2} W^{*}$ induces a map $\varphi: F \widehat{\otimes} \Lambda^{k} W^{*} \rightarrow \Lambda^{k+2} W^{*}$ for any $k$. We will assume that the rank of these maps is constant on $X$, for any $k$. In particular, $\varphi\left(F \widehat{\otimes} \Lambda^{k} W^{*}\right)$ forms a vector subbundle of $\Lambda^{k+2} W^{*}$ on $X$ for any $k$. Set $F_{\varphi}:=\varphi\left(F \widehat{\otimes} \Lambda W^{*}\right)$ and let $F_{\varphi, \perp}$ be the orthogonal complement of $F_{\varphi}$ in $\Lambda W^{*}$ over $X$.

By construction, we thus have an orthogonal decomposition

$$
\Lambda W^{*}=F_{\varphi} \oplus F_{\varphi, \perp}
$$

and by (1.12) and (1.16), this decomposition induces (1.14), that is

$$
\begin{aligned}
\mathcal{J} / \mathcal{I} & =\mathscr{C}^{\infty}\left(X, F_{\varphi}\right), \\
\mathcal{Q} & =\mathscr{C}^{\infty}\left(X, F_{\varphi, \perp}\right) .
\end{aligned}
$$

We denote by $\pi_{F_{\varphi}}$ and $\pi_{F_{\varphi, \perp}}$ the orthogonal projections from $\Lambda W^{*}$ onto $F_{\varphi}$ and $F_{\varphi, \perp}$. In order to make computations with the operators on $\mathcal{Q}$, we construct intermediate operators on $\Lambda W^{*}$. First we define

$$
d_{W}:=\pi_{W} \circ d \circ \pi_{W}: \Lambda W^{*} \rightarrow \Lambda W^{*},
$$

where $\pi_{W}$ is the projection from $\Lambda\left(T^{*} X\right)$ on $\Lambda W^{*}$ in the decomposition (1.13). Beware that there is no reason for $d_{W}^{2}$ to be 0 . The adjoint $d_{W}^{*}$ of $d_{W}$ satisfies

$$
d_{W}^{*}=\pi_{W} \circ d^{*} \circ \pi_{W}
$$

From (1.1), (1.18) and (1.19), we have

$$
\begin{aligned}
& d_{\mathcal{Q}}=\pi_{\mathcal{Q}} \circ d_{W} \circ \pi_{\mathcal{Q}}, \\
& d_{\mathcal{Q}}^{*}=\pi_{\mathcal{Q}} \circ d_{W}^{*} \circ \pi_{\mathcal{Q}} .
\end{aligned}
$$

By (1.18) and (1.19),$d_{W}$ and $d_{W}^{*}$ are first order differential operators on $\Lambda W^{*}$ and their principal symbols are, for $\xi \in T^{*} X$

$$
\begin{aligned}
& \sigma_{1}\left(d_{W}, \xi\right)=\sqrt{-1} \xi_{W} \wedge, \\
& \sigma_{1}\left(d_{W}^{*}, \xi\right)=-\sqrt{-1} i_{\xi_{W}^{*}},
\end{aligned}
$$

where $\xi_{W}$ is the orthogonal projection of $\xi$ on $W^{*}$ and $\xi_{W}^{*} \in W$ is the metric dual of $\xi_{W}$. 
By (1.17) and (1.20),$d_{\mathcal{Q}}$ and $d_{\mathcal{Q}}^{*}$ are first order differential operators on $F_{\varphi, \perp}$ and their principal symbols are

$$
\begin{aligned}
& \sigma_{1}\left(d_{\mathcal{Q}}, \xi\right)=\sqrt{-1} \pi_{F_{\varphi, \perp}} \xi_{W} \wedge \pi_{F_{\varphi, \perp}}, \\
& \sigma_{1}\left(d_{\mathcal{Q}}^{*}, \xi\right)=-\sqrt{-1} \pi_{F_{\varphi, \perp}} i_{\xi_{W}^{*}} \pi_{F_{\varphi, \perp}} .
\end{aligned}
$$

One also gets the adjoint formula of (1.2)

$$
\pi_{\mathcal{Q}} \circ d^{*} \circ \pi_{\mathcal{Q}}=d^{*} \circ \pi_{\mathcal{Q}}
$$

Taking the principal symbols of (1.2) and (1.23), we have

$$
\begin{array}{r}
\pi_{F_{\varphi, \perp}} \xi_{W} \wedge \pi_{F_{\varphi, \perp}}=\pi_{F_{\varphi, \perp}} \xi_{W} \wedge \\
\pi_{F_{\varphi, \perp}} i_{\xi_{W}^{*}} \pi_{F_{\varphi, \perp}}=i_{\xi_{W}^{*}} \pi_{F_{\varphi, \perp}} .
\end{array}
$$

Proposition 1.7. The characteristic Laplacian is a second order differential operator on $F_{\varphi, \perp}$ and its principal symbol is

$$
\sigma_{2}\left(\Delta_{\mathcal{Q}}, \xi\right)=\pi_{F_{\varphi, \perp}}\left(\left|\xi_{W}\right|^{2}-i_{\xi_{W}^{*}} \pi_{F_{\varphi}} \xi_{W} \wedge\right) \pi_{F_{\varphi, \perp}}
$$

Remark 1.8. The first term in (1.25) is the term suggested in [8] but the second term was forgotten. Because of this second term, one cannot apply Hörmander's condition of hypoellipticity; see Example 1.9.

Proof of Proposition 1.7. By (1.5), (1.22) and (1.24), $\Delta_{\mathcal{Q}}$ is a second order differential operator on $F_{\varphi, \perp}$ and its principal symbol is

$$
\begin{aligned}
\sigma_{2}\left(\Delta_{\mathcal{Q}}, \xi\right) & =\pi_{F_{\varphi, \perp}} \xi_{W} \wedge \pi_{F_{\varphi, \perp}} i_{\xi_{W}^{*}} \pi_{F_{\varphi, \perp}}+\pi_{F_{\varphi, \perp}} i_{\xi_{W}^{*}} \pi_{F_{\varphi, \perp}} \xi_{W} \wedge \pi_{F_{\varphi, \perp}} \\
& =\pi_{F_{\varphi, \perp}} \xi_{W} \wedge i_{\xi_{W}^{*}} \pi_{F_{\varphi, \perp}}+\pi_{F_{\varphi, \perp}} i_{\xi_{W}^{*}} \xi_{W} \wedge \pi_{F_{\varphi, \perp}}-\pi_{F_{\varphi, \perp}} i_{\xi_{W}^{*}} \pi_{F_{\varphi}} \xi_{W} \wedge \pi_{F_{\varphi, \perp}} \\
& =\pi_{F_{\varphi, \perp}}\left(\left|\xi_{W}\right|^{2}-i_{\xi_{W}^{*}} \pi_{F_{\varphi}} \xi_{W} \wedge\right) \pi_{F_{\varphi, \perp}} .
\end{aligned}
$$

In the second equality, we use (1.24) and in the third, we use the identity

$$
i_{\xi_{W}^{*}} \xi_{W} \wedge+\xi_{W} \wedge i_{\xi_{W}^{*}}=\left|\xi_{W}\right|^{2}
$$

The proof of Proposition 1.7 is completed.

Example 1.9. We review Example 1.5 and compute the principal symbol of the characteristic Laplacian in degree one. With the identifications at the beginning of the paragraph, $\mathcal{Q}^{1}$ is the space of sections of $W^{*}$ and $\mathcal{Q}^{2}$ is zero. Let $\eta$ be in $W^{*}$. Since in (1.25), only the projection $\xi_{W}$ of $\xi$ is involved, we can restrict the symbol to the $\xi$ belonging to $W^{*}$. We have

$$
\begin{aligned}
\sigma_{2}\left(\Delta_{\mathcal{Q}}, \xi\right) \eta & =|\xi|^{2} \eta-\pi_{F_{\varphi, \perp}} i_{\xi^{*}} \pi_{F_{\varphi}}(\xi \wedge \eta) \\
& =|\xi|^{2} \eta-i_{\xi^{*}}(\xi \wedge \eta) \\
& =|\xi|^{2} \eta-\left(|\xi|^{2} \eta-\eta\left(\xi^{*}\right) \xi\right) \\
& =\eta\left(\xi^{*}\right) \xi .
\end{aligned}
$$

In the second equality, we use that $F_{\varphi, \perp}$ (resp. $F_{\varphi}$ ) equals $\Lambda W^{*}$ in degree 1 (resp. 2).

If $\xi=\left(\xi_{1}, \xi_{2}\right)^{t}$ and $\eta=\left(\eta_{1}, \eta_{2}\right)^{t}$ in an orthonormal frame for $W^{*}$, we get

$$
\sigma_{2}\left(\Delta_{\mathcal{Q}}, \xi\right)\left(\begin{array}{l}
\eta_{1} \\
\eta_{2}
\end{array}\right)=\left(\xi_{1} \eta_{1}+\xi_{2} \eta_{2}\right)\left(\begin{array}{l}
\xi_{1} \\
\xi_{2}
\end{array}\right)=\left(\begin{array}{l}
\xi_{1}^{2} \eta_{1}+\xi_{1} \xi_{2} \eta_{2} \\
\xi_{1} \xi_{2} \eta_{1}+\xi_{2}^{2} \eta_{2}
\end{array}\right)
$$


Otherwise said, we have the equality

$$
-\Delta_{\mathcal{Q}}\left(\begin{array}{l}
\eta_{1} \\
\eta_{2}
\end{array}\right)=\left(\begin{array}{c}
X_{1}^{2} \eta_{1}+X_{1} X_{2} \eta_{2} \\
X_{1} X_{2} \eta_{1}+X_{2}^{2} \eta_{2}
\end{array}\right)+X_{0}\left(\begin{array}{l}
\eta_{1} \\
\eta_{2}
\end{array}\right)
$$

where $\left(X_{1}, X_{2}\right)$ is a local frame of $W$ and $X_{0}$ is a first order differential operator, which does not necessarily act componentwise. Since the second order does not act componentwise, one cannot apply Hörmander's condition of Theorem 1.4.

1.4. The complex situation. In the remainder of the article, we will be interested in Pfaffian systems over a complex manifold. More precisely, let $(X, J)$ be a compact complex manifold; $J$ induces a splitting $T X \otimes_{\mathbb{R}} \mathbb{C}=T^{(1,0)} X \oplus T^{(0,1)} X$, where $T^{(1,0)} X$ and $T^{(0,1)} X$ are the eigenbundles of $J$ corresponding to the eigenvalues $\sqrt{-1}$ and $-\sqrt{-1}$, respectively. Let $T^{*(1,0)} X$ and $T^{*(0,1)} X$ be the corresponding dual bundles.

We still denote by $\Omega^{k}(X)$ the space of smooth $k$-forms on $X$ with values in $\mathbb{C}$. Let

$$
\Lambda^{p, q}\left(T^{*} X\right)=\Lambda^{p}\left(T^{*(1,0)} X\right) \otimes \Lambda^{q}\left(T^{*(0,1)} X\right), \quad \Omega^{p, q}(X):=\mathscr{C}^{\infty}\left(X, \Lambda^{p, q}\left(T^{*} X\right)\right) .
$$

Then $\Omega^{p, q}(X)$ is the space of smooth $(p, q)$-forms on $X$, and $\Omega^{k}(X)=\oplus_{p+q=k} \Omega^{p, q}(X)$.

Let $\Theta$ be a real $(1,1)$-form such that

$$
g^{T X}(\cdot, \cdot)=\Theta(\cdot, J \cdot)
$$

defines a Riemannian metric on $T X$. The triple $(X, J, \Theta)$ is called a complex Hermitian manifold. If $\Theta$ is a closed form, then the form $\Theta$ is called a Kähler form on $X$.

We denote the holomorphic tangent bundle by $T_{h} X$. Let $W \subset T_{h} X$ be a (constantrank) holomorphic distribution. We consider the Pfaffian system associated to the distribution $W_{\mathbb{R}}$. Otherwise said, if we denote by $F \subset T_{h}^{*} X$ the holomorphic annihilator of $W$, then the exterior differential system $\mathcal{J}$ we consider is generated by $F_{\mathbb{R}} \subset T^{*} X$. Beware of the notations that differ from the real case. Remark that $\mathcal{J}$ is not only $d$-stable: it is also $\partial$ and $\bar{\partial}$-stable. Indeed, if $I_{X}=\mathscr{C}^{\infty}(X, F)$ is the space of smooth sections of $F$ on $X$ then $\bar{I}_{X}=\mathscr{C}^{\infty}(X, \bar{F})$ and $d$ acts on $I_{X}$ (resp. $\bar{I}_{X}$ ) as $\partial$ modulo $\bar{I}_{X} \cdot \Omega^{1}(X)$ (resp. $\bar{\partial}$ modulo $\left.I_{X} \cdot \Omega^{1}(X)\right)$.

Locally, if $\left(\theta_{j}\right)$ is a holomorphic frame of $F$, the forms in $\mathcal{I}$ can be written as

$$
\sum_{j} \theta_{j} \wedge \psi_{j}+\bar{\theta}_{j} \wedge \phi_{j}
$$

where $\psi_{j}, \phi_{j}$ are arbitrary forms, and those in $\mathcal{J}$ are of the form

$$
\sum_{j} \theta_{j} \wedge \psi_{j}+\bar{\theta}_{j} \wedge \phi_{j}+d \theta_{j} \wedge \omega_{j}+d \bar{\theta}_{j} \wedge \chi_{j}
$$

where $\psi_{j}, \phi_{j}, \omega_{j}, \chi_{j}$ are arbitrary forms.

We still denote by $\mathcal{Q}$ the orthogonal of $\mathcal{J}$. Besides the operator $d_{\mathcal{Q}}$, we also define $\partial_{\mathcal{Q}}$ and $\bar{\partial}_{\mathcal{Q}}$ by

$$
\partial_{\mathcal{Q}}:=\pi_{\mathcal{Q}} \circ \partial \circ \pi_{\mathcal{Q}}, \quad \bar{\partial}_{\mathcal{Q}}:=\pi_{\mathcal{Q}} \circ \bar{\partial} \circ \pi_{\mathcal{Q}}
$$

Moreover, as in (1.4), the adjoints of $\partial_{\mathcal{Q}}$ and $\bar{\partial}_{\mathcal{Q}}$ (for the natural $L^{2}$-structure on $\mathcal{Q}$ ) are the restrictions to $\mathcal{Q}$ of $\partial^{*}, \bar{\partial}^{*}$ the adjoints of $\partial$ and $\bar{\partial}$.

If we denote by $N$ the orthogonal complement of $W$ in $\left(T X, g^{T X}\right)$, one obtains analogue decompositions as the ones in the previous subsection. In particular, denoting by $\mathcal{I}$ the 
algebraic ideal generated by $F_{\mathbb{R}}$, one has the analogues of (1.11), (1.12), (1.13) and (1.14):

$$
\begin{aligned}
& \mathcal{I}=\mathscr{C}^{\infty}\left(X, N_{\mathbb{R}}^{*} \widehat{\otimes} \Lambda\left(T^{*} X\right)\right), \\
& \Omega_{W}(X):=\mathscr{C}^{\infty}\left(X, \Lambda W_{\mathbb{R}}^{*}\right), \\
& \Omega_{(X)}=\mathcal{I} \oplus \Omega_{W}(X), \\
& \Omega_{W}(X)=\mathcal{J} / \mathcal{I} \oplus \mathcal{Q} .
\end{aligned}
$$

All of these spaces carry a natural bigrading. Denoting by $\pi_{W}$ the orthogonal projection from $\Lambda\left(T^{*} X\right)$ onto $\Lambda W_{\mathbb{R}}^{*}$, we define

$$
\begin{array}{cl}
\partial_{W}:=\pi_{W} \circ \partial \circ \pi_{W}, & \bar{\partial}_{W}:=\pi_{W} \circ \bar{\partial} \circ \pi_{W}, \\
\partial_{W}^{*}=\pi_{W} \circ \partial^{*} \circ \pi_{W}, & \bar{\partial}_{W}^{*}=\pi_{W} \circ \bar{\partial}^{*} \circ \pi_{W} .
\end{array}
$$

Then, we have, besides equations (1.18), (1.19) and (1.20),

$$
\begin{array}{ll}
\partial_{\mathcal{Q}}=\pi_{\mathcal{Q}} \circ \partial_{W} \circ \pi_{\mathcal{Q}}, & \bar{\partial}_{\mathcal{Q}}=\pi_{\mathcal{Q}} \circ \bar{\partial}_{W} \circ \pi_{\mathcal{Q}}, \\
\partial_{\mathcal{Q}}^{*}=\pi_{\mathcal{Q}} \circ \partial_{W}^{*} \circ \pi_{\mathcal{Q}}, & \bar{\partial}_{\mathcal{Q}}^{*}=\pi_{\mathcal{Q}} \circ \bar{\partial}_{W}^{*} \circ \pi_{\mathcal{Q}} .
\end{array}
$$

We still define a map $\varphi: F \rightarrow \Lambda^{2} W^{*}$ as in (1.15). Since in the definition (1.15), we can take $\theta, v, w$ to be holomorphic sections, it proves that $\varphi$ is a holomorphic map. We still assume that the induced map $\varphi: F \widehat{\otimes} \Lambda W_{\mathbb{R}}^{*} \rightarrow \Lambda W_{\mathbb{R}}^{*}$ has constant rank. Set $F_{\varphi}:=\varphi\left(F \widehat{\otimes} \Lambda W^{*}\right)$ and $F_{\varphi, \perp}$ the orthogonal complement of $F_{\varphi}$ in $\Lambda W^{*}$ and let $\pi_{F_{\varphi},} \pi_{F_{\varphi, \perp}}$ be the orthogonal projections from $\Lambda W^{*}$ onto $F_{\varphi}, F_{\varphi, \perp}$.

Then $F_{\varphi} \widehat{\otimes} \Lambda \bar{W}^{*}+\Lambda W^{*} \widehat{\otimes} \bar{F}_{\varphi}$ and $F_{\varphi, \perp} \widehat{\otimes} \bar{F}_{\varphi, \perp}$ are vector subbundles of $\Lambda W_{\mathbb{R}}^{*} \otimes_{\mathbb{R}} \mathbb{C}$ over $X$, and

$$
\Lambda W_{\mathbb{R}}^{*} \otimes_{\mathbb{R}} \mathbb{C}=\left(F_{\varphi} \widehat{\otimes} \Lambda \bar{W}^{*}+\Lambda W^{*} \widehat{\otimes} \bar{F}_{\varphi}\right) \oplus F_{\varphi, \perp} \widehat{\otimes} \bar{F}_{\varphi, \perp}
$$

In this decomposition, we have, as in (1.17),

$$
\begin{aligned}
\mathcal{J} / \mathcal{I} & =\mathscr{C}^{\infty}\left(X, F_{\varphi} \widehat{\otimes} \Lambda \bar{W}^{*}+\Lambda W^{*} \widehat{\otimes} \bar{F}_{\varphi}\right) . \\
\mathcal{Q} & =\mathscr{C}^{\infty}\left(X, F_{\varphi, \perp} \widehat{\otimes} \bar{F}_{\varphi, \perp}\right) .
\end{aligned}
$$

Example 1.10. Example 1.5 can also be seen in the complex situation but an interesting phenomenon appears in degree 1 . Consider the complex manifold $M=\mathbb{C}^{3}$ with complex coordinates $(x, u, p)$ and the holomorphic 1 -form $\theta=d u-p d x$. We denote by $(\cdot, \cdot)$ a Hermitian metric on $M$ and by $|\cdot|$ the corresponding norm. Using the notations of this subsection, $W$ is a holomorphic vector subbundle of $T_{h} X$ of $\operatorname{rank} 2$. Hence $\Lambda^{2} W^{*}$ is of rank 1 and we have $F_{\varphi}=\Lambda^{2} W^{*}$. We thus obtain the following bidegree decomposition of $\mathcal{Q}$ over an open set $U$ :

- $\mathcal{Q}^{0,0}(U)=\mathscr{C}^{\infty}(U)$,

- $\mathcal{Q}^{1,0}(U)=\mathscr{C}^{\infty}\left(U, W^{*}\right)$,

- $\mathcal{Q}^{0,1}(U)=\mathscr{C}^{\infty}\left(U, \bar{W}^{*}\right)$,

- $\mathcal{Q}^{1,1}(U)=\mathscr{C}^{\infty}\left(U, W^{*} \widehat{\otimes} \bar{W}^{*}\right)$.

Take $\left(X_{1}, X_{2}\right)$ a holomorphic frame of $W$ on $\bar{U}$ and $\left(\alpha^{1}, \alpha^{2}\right)$ its dual frame. We study the smoothness of harmonic forms for $\Delta_{\mathcal{Q}}$ in degrees 0,1 and 2 .

For $f \in L^{2}(U, \mathbb{C})$, one has $\Delta_{\mathcal{Q}} f=0$ if and only if $d_{\mathcal{Q}} f=0$. Using the frames, one obtains

$$
d_{\mathcal{Q}} f=X_{1}(f) \alpha^{1}+X_{2}(f) \alpha^{2}+\bar{X}_{1}(f) \bar{\alpha}^{1}+\bar{X}_{2}(f) \bar{\alpha}^{2} .
$$


Since $X_{1}, X_{2}, \bar{X}_{1}$ and $\bar{X}_{2}$ generate by brackets the tangent bundle of $U$, a function $f$ such that $d_{\mathcal{Q}} f=0$ is in fact locally constant. Remark that the same argument works for any distribution $W$ which is bracket-generating. This proves that the harmonic functions are smooth.

In degree 1 , let $\mu=\mu_{1} \alpha^{1}+\mu_{2} \alpha^{2}$ be in $L^{2}\left(U, W^{*}\right)$ (here $\mu_{1}, \mu_{2} \in L^{2}(U, \mathbb{C})$ ). Such a form is cancelled by $\Delta_{\mathcal{Q}}^{1}$ if and only if it is cancelled by both $d_{\mathcal{Q}}$ and $d_{\mathcal{Q}}^{*}$.

We denote by $\operatorname{div}(Y)$ the divergence of a vector field $Y$ and by $d v_{X}$ the Riemannian volume form on $\left(X, g^{T X}\right)$.

Lemma 1.11. The following identities hold:

$$
\begin{aligned}
d_{\mathcal{Q}} \mu & =\sum_{i, j=1}^{2} \bar{X}_{j}\left(\mu_{i}\right) \bar{\alpha}^{j} \wedge \alpha^{i} \\
d_{\mathcal{Q}}^{*} \mu & =-\operatorname{div}\left(\sum_{i=1}^{2}\left(\mu, \alpha_{i}\right) \overline{X_{i}}\right) .
\end{aligned}
$$

Proof. Since $\mathcal{Q}^{2,0}(U)=0$, we know $d_{\mathcal{Q}} \mu=\bar{\partial}_{\mathcal{Q}} \mu$. The 2 -form $d \alpha^{k}$ satisfies

$$
d \alpha^{k}\left(Y_{1}, Y_{2}\right)=Y_{1}\left(\alpha^{k}\left(Y_{2}\right)\right)-Y_{2}\left(\alpha^{k}\left(Y_{1}\right)\right)-\alpha^{k}\left(\left[Y_{1}, Y_{2}\right]\right) .
$$

Since $\left(\alpha^{1}, \alpha^{2}\right)$ is the dual basis of $\left(X_{1}, X_{2}\right)$, this simplifies to $d \alpha^{k}\left(Y_{1}, Y_{2}\right)=-\alpha^{k}\left(\left[Y_{1}, Y_{2}\right]\right)$ when $Y_{l}$ is either a $X_{i}$ or a $\bar{X}_{j}$. Since the $X_{i}$ are holomorphic, the brackets $\left[X_{i}, \bar{X}_{j}\right]$ vanish and $d \alpha^{k}\left(X_{i}, \bar{X}_{j}\right)=0$. This implies that

$$
d_{\mathcal{Q}} \mu=\sum_{i=1}^{2} \bar{\partial}_{\mathcal{Q}} \mu_{i} \alpha^{i}=\sum_{i, j=1}^{2} \bar{X}_{j}\left(\mu_{i}\right) \bar{\alpha}^{j} \wedge \alpha^{i} .
$$

For the second equality, one has for every smooth function $f$,

$$
\begin{aligned}
\left(d_{\mathcal{Q}}^{*} \mu, f\right) & =\left(\partial_{\mathcal{Q}}^{*} \mu, f\right) \\
& =\left(\mu, \partial_{\mathcal{Q}} f\right) \\
& =-\sum_{i=1}^{2} \int_{M} \overline{X_{i}(f)}\left(\mu, \alpha^{i}\right) d v_{X} \\
& =-\sum_{i=1}^{2} \int_{M} \bar{f}\left(\bar{X}_{i}\left(\mu, \alpha^{i}\right)+\operatorname{div} \bar{X}_{i}\right) d v_{X} .
\end{aligned}
$$

Hence $d_{\mathcal{Q}}^{*} \mu=-\sum_{i=1}^{2}\left(\bar{X}_{i}\left(\mu, \alpha^{i}\right)+\left(\mu, \alpha^{i}\right) \operatorname{div} \bar{X}_{i}\right)=-\operatorname{div}\left(\sum_{i=1}^{2}\left(\mu, \alpha_{i}\right) \overline{X_{i}}\right)$.

Using Lemma 1.11, if $\Delta_{\mathcal{Q}}^{1} \mu=0$ then $\bar{X}_{j}\left(\mu_{i}\right)=0$ for all $i, j$ from 1 to 2. Since the $\bar{X}_{j}$ generate by brackets the anti-holomorphic tangent bundle, this is equivalent to $\mu_{i}$ being holomorphic in the weak sense. Since $\bar{\partial}$ is an elliptic operator on functions, the $\mu_{i}$ are holomorphic. This shows that the harmonic forms of bidegree $(1,0)$ (resp. $(0,1))$ are holomorphic (resp. anti-holomorphic) sections of $W^{*}$ (resp. $\bar{W}^{*}$ ). In particular, they are smooth, contrary to what happened in the real setting.

In degree 2 , consider a form $\nu=\sum_{i, j=1}^{2} \nu_{i j} \bar{\alpha}^{j} \wedge \alpha^{i}$ in $L^{2}\left(U, W^{*} \widehat{\otimes} \bar{W}^{*}\right)$. It is cancelled by $\Delta_{\mathcal{Q}}^{2}$ if and only if $d_{\mathcal{Q}}^{*} \nu=0$. 
Lemma 1.12. The 2-form $\nu$ is harmonic if and only if for $k=1,2$,

$$
\begin{aligned}
& \operatorname{div}\left(\sum_{l=1}^{2}\left(\nu, \bar{\alpha}^{l} \wedge \alpha^{k}\right) X_{l}\right)=0, \\
& \operatorname{div}\left(\sum_{l=1}^{2}\left(\nu, \bar{\alpha}^{k} \wedge \alpha^{l}\right) \bar{X}_{l}\right)=0 .
\end{aligned}
$$

Proof. We just show that the first equation is equivalent to the vanishing of $\bar{\partial}_{\mathcal{Q}}^{*} \nu$. For every $(1,0)$-form $\mu=\mu_{1} \alpha^{1}+\mu_{2} \alpha^{2}$,

$$
\begin{aligned}
\left(\bar{\partial}_{\mathcal{Q}}^{*} \nu, \mu\right) & =\left(\nu, \bar{\partial}_{\mathcal{Q}} \mu\right) \\
& =\left(\nu, \sum_{k, l=1}^{2} \bar{X}_{l}\left(\mu_{k}\right) \bar{\alpha}^{l} \wedge \alpha^{k}\right) \text { by Lemma 1.11 } \\
& =\sum_{k, l=1}^{2} \int_{M} X_{l}\left(\bar{\mu}_{k}\right)\left(\nu, \bar{\alpha}^{l} \wedge \alpha^{k}\right) d v_{X} \\
& =-\sum_{k, l=1}^{2} \int_{M} \bar{\mu}_{k} \operatorname{div}\left(\left(\nu, \bar{\alpha}^{l} \wedge \alpha^{k}\right) X_{l}\right) d v_{X}
\end{aligned}
$$

This is zero for all $\mu$ if and only if $\operatorname{div}\left(\sum_{l=1}^{2}\left(\nu, \bar{\alpha}^{l} \wedge \alpha^{k}\right) X_{l}\right)$ is 0 for $k=1,2$.

It seems difficult to unravel these equations in general. We will only consider two different choices of the metric.

Standard metric. In the particular case where the metric on $\mathbb{C}^{3}$ is the standard one, one can choose for $X_{1}, X_{2}$ orthogonal holomorphic vectors with zero divergence (take $X_{1}=\frac{\partial}{\partial p}$ and $X_{2}=p \frac{\partial}{\partial u}+\frac{\partial}{\partial x}$. Moreover, $X_{1}$ has norm 1. Then the equations of Lemma 1.12 become

$$
\begin{aligned}
& \sum_{l} X_{l}\left(\nu_{l k}\left|\bar{\alpha}^{l} \wedge \alpha^{k}\right|^{2}\right)=0, \\
& \sum_{l} \bar{X}_{l}\left(\nu_{k l}\left|\bar{\alpha}^{k} \wedge \alpha^{l}\right|^{2}\right)=0
\end{aligned}
$$

where $\nu=\nu_{l k} \bar{\alpha}^{l} \wedge \alpha^{k}$. One can take $\nu_{12}=\nu_{21}=\nu_{22}=0$. Then the equations are simply $X_{1} \nu_{11}=\bar{X}_{1} \nu_{11}=0$. Since $X_{1}$ is holomorphic, $\left[X_{1}, \bar{X}_{1}\right]=0$ and, by Frobenius theorem, one can choose $\nu_{11}$ constant in the directions of $X_{1}$ and $\bar{X}_{1}$ but $\nu_{11}$ not smooth. This shows the existence of non-smooth harmonic 2-forms.

Heisenberg metric. Consider the case where we see $\mathbb{C}^{3}$ as the complex Heisenberg group (see Example 1.5), endowed with a right-invariant Hermitian metric and with a right-invariant contact form. Choose a basis $\left(X_{1}^{\prime}, X_{2}^{\prime}\right)$ of $W$ at the identity $e$ of $\mathcal{H}^{3}$. Consider the corresponding right-invariant vector fields on $\mathcal{H}^{3}$, denoted by $\widetilde{X_{1}^{\prime}}$ and $\widetilde{X_{2}^{\prime}}$. Since these vector fields and the volume form are right-invariant, the divergences of $\widetilde{X_{1}^{\prime}}$ and $\widetilde{X_{2}^{\prime}}$ are constant. Hence, to certain linear combination $X_{1}$ of $X_{1}^{\prime}$ and $X_{2}^{\prime}$ corresponds a right-invariant vector field with zero divergence. We can moreover assume that $X_{1}$ is a unit vector and complete it to an orthonormal basis $\left(X_{1}, X_{2}\right)$ of $W_{e}$. Thus we get a 
holomorphic orthonormal frame $\left(\widetilde{X_{1}}, \widetilde{X_{2}}\right)$ of $W$ and $\widetilde{X_{1}}$ has zero divergence. Then, the same argument as above shows the existence of non-smooth harmonic 2-forms.

Remark 1.13. This last example with the Heisenberg metric is in fact the prototype of the situation described in Conjecture 0.1. Indeed, take $\Gamma$ a cocompact subgroup of the complex Heisenberg group. Then, by invariance of the metric and of the distribution, we obtain a counterexample to the hypoellipticity of the characteristic Laplacian in a compact complex contact 3-manifold. Those manifolds are the simplest examples of period domains, with non-trivial Griffith's transversality condition (that is, with a distribution not equal to the whole tangent space).

\section{Answer to Question 0.3}

The aim of this section is to prove the following theorem, which is an answer to question 0.3 ;

Theorem 2.1. In the notations of Question 0.3 and Section 1, the characteristic Laplacian never respects the bigrading on $\mathcal{Q}^{\bullet}$ when the distribution $W$ is not involutive.

This section is organized as follows. In Subsection 2.1, we show that a complex Hermitian manifold is Kähler if and only if the Hodge Laplacian preserves the bigrading on $\Omega(X)$. In Subsection 2.2, we establish a generalized sub-Kähler identity. In Subsection 2.3, we establish Theorem 2.1.

2.1. The classical case. First we study the case where the distribution $W$ is the whole tangent space $T X$, which is interesting for itself. We thus have $\mathcal{Q}=\Omega(X)$ and the characteristic Laplacian is the usual Hodge Laplacian, which we simply denote by $\Delta$. Remark that Theorem 2.1 says nothing in this case.

It is well known that for a Kähler manifold, its Hodge Laplacian preserves the bigrading of the differential forms (cf. [9, §0.7], [12, Corollary 1.4.13]). This implies the decomposition of the complex valued de-Rham cohomology in bidegree type for a compact Kähler manifold; this was in fact the initial interest of the authors for the general question 0.3. In [8, §III. A], Green, Griffiths and Kerr claimed that Chern [6] proved that for Hermitian manifolds, if its Hodge Laplacian preserves the bigrading of the differential forms, then the Hermitian metric is Kähler. After communications with Professors Bryant and Griffiths, we realized that Chern did not claim this result in his paper [6], and it seems that one could not find a proof in the literature.

Theorem 2.2. The complex Hermitian manifold $(X, J, \Theta)$ is Kähler if and only if $\Delta$ preserves the bigrading on $\Omega(X)$, i.e., $\Delta$ sends $(p, q)$-forms to $(p, q)$-forms.

We first introduce some notations from [12].

For any $\mathbb{Z}_{2}$-graded vector space $V=V^{+} \oplus V^{-}$, the natural $\mathbb{Z}_{2}$-grading on $\operatorname{End}(V)$ is defined by

$$
\operatorname{End}(V)^{+}=\operatorname{End}\left(V^{+}\right) \oplus \operatorname{End}\left(V^{-}\right), \quad \operatorname{End}(V)^{-}=\operatorname{Hom}\left(V^{+}, V^{-}\right) \oplus \operatorname{Hom}\left(V^{-}, V^{+}\right),
$$

and we define $\operatorname{deg} B=0$ for $B \in \operatorname{End}(V)^{+}$, and $\operatorname{deg} B=1$ for $B \in \operatorname{End}(V)^{-}$. For $B, C \in \operatorname{End}(V)$, we define their supercommutator (or graded Lie bracket) by

$$
[B, C]=B C-(-1)^{\operatorname{deg} B \cdot \operatorname{deg} C} C B \text {. }
$$


Then for $B, B^{\prime}, C \in \operatorname{End}(V)$, the Jacobi identity holds:

$$
\begin{aligned}
(-1)^{\operatorname{deg} C \cdot \operatorname{deg} B^{\prime}\left[B^{\prime},[B, C]\right]+(-1)^{\operatorname{deg} B^{\prime} \cdot \operatorname{deg} B}\left[B,\left[C, B^{\prime}\right]\right]} \\
+(-1)^{\operatorname{deg} B \cdot \operatorname{deg} C}\left[C,\left[B^{\prime}, B\right]\right]=0 .
\end{aligned}
$$

We will apply the above notation for $\Omega^{\bullet}(X)$ with natural $\mathbb{Z}_{2}$-grading induced by the parity of the degree, (cf. [12, (1.3.31)]).

We define the Lefschetz operator $L=\Theta \wedge$ on $\Lambda^{\bullet \bullet \bullet}\left(T^{*} X\right)$ and its adjoint $\Lambda=i(\Theta)$ with respect to the Hermitian product $\langle\cdot, \cdot\rangle_{\Lambda} \bullet \bullet \bullet$ induced by $g^{T X}$. For $\left\{w_{j}\right\}_{j=1}^{m}$ a local orthonormal frame of $T^{(1,0)} X$, we have

$$
L=\sqrt{-1} \sum_{j=1}^{m} w^{j} \wedge \bar{w}^{j} \wedge, \quad \Lambda=-\sqrt{-1} \sum_{j=1}^{m} i_{\bar{w}_{j}} i_{w_{j}},
$$

where $\wedge$ and $i$ denote the exterior and interior product, respectively. The Hermitian torsion operator is defined by

$$
\mathcal{T}:=[\Lambda, \partial \Theta]=[i(\Theta), \partial \Theta] .
$$

Proof of Theorem 2.2. If $(X, J, \Theta)$ is Kähler, then it is a classical result that $\Delta$ preserves the bigrading on $\Omega^{\bullet}(X)$, cf. for example [12, Corollary 1.4.13] for a proof.

We assume now that $\Delta$ preserves the bigrading on $\Omega^{\bullet}(X)$.

Let $\square:=\partial \partial^{*}+\partial^{*} \partial ; \square:=\bar{\partial} \bar{\partial}^{*}+\bar{\partial}^{*} \bar{\partial}$ be the usual $\partial$-Laplacian and $\bar{\partial}$-Laplacian. Then as $d=\partial+\bar{\partial}$ and $d^{2}=0$, we have (cf. [12, 1.4.50)])

$$
\Delta=\left[d, d^{*}\right]=\left[\partial+\bar{\partial}, \partial^{*}+\bar{\partial}^{*}\right]=\square+\bar{\square}+\left[\partial, \bar{\partial}^{*}\right]+\left[\bar{\partial}, \partial^{*}\right] .
$$

As $\square, \square$ preserve the bigrading on $\Omega^{\bullet}(X)$, and $\left[\partial, \bar{\partial}^{*}\right]: \Omega^{\bullet \bullet \bullet}(X) \rightarrow \Omega^{\bullet+1, \bullet-1}(X)$, we know that $\Delta$ preserves the bigrading on $\Omega^{\bullet}(X)$ if and only if

$$
\left[\partial, \bar{\partial}^{*}\right]=0 .
$$

By the generalized Kähler identities [12, (1.4.38d)] (cf. [7]) for $E=\mathbb{C}$ therein, we get

$$
[\Lambda, \partial]=\sqrt{-1}\left(\bar{\partial}^{*}+\overline{\mathcal{T}}^{*}\right)
$$

From (2.7), we get

$$
\left[\partial, \bar{\partial}^{*}\right]=-\sqrt{-1}[\partial,[\Lambda, \partial]]+\left[\partial, \overline{\mathcal{T}}^{*}\right]
$$

But by (2.2), we get

$$
[\partial,[\Lambda, \partial]]=[\Lambda,[\partial, \partial]]+[\partial,[\partial, \Lambda]]
$$

As $[\partial, \partial]=2 \partial^{2}=0$ and $[\partial, \Lambda]=-[\Lambda, \partial]$, we get from (2.9) that

$$
[\partial,[\Lambda, \partial]]=0 \text {. }
$$

From (2.8) and (2.10), we know that (2.6) is equivalent to

$$
\left[\bar{\partial}^{*}, \mathcal{T}\right]=0 \text {. }
$$


By [12, (1.4.9)], the operator $\bar{\partial}^{*}$ has the form $\bar{\partial}^{*}=-\sum_{j} i_{\bar{w}_{j}} \widetilde{\nabla}_{w_{j}}^{T X}+0$-order terms, here $\widetilde{\nabla}^{T X}$ is certain connection on $\Lambda\left(T^{*} X\right)$, thus $\left[\bar{\partial}^{*}, \mathcal{T}\right]$ is a first order differential operator, and its principal symbol $\sigma$ is: for $\xi \in T^{*} X$,

$$
\sigma(\xi)=-\sqrt{-1} \sum_{j}\left(\xi, w_{i}\right) \cdot\left[i_{\bar{w}_{i}}, \mathcal{T}\right]
$$

By [12, Lemma 1.4.10],

$$
\mathcal{T}=-\frac{\sqrt{-1}}{2} \sum_{j k l}(\partial \Theta)\left(w_{j}, w_{k}, \bar{w}_{l}\right)\left[2 w^{k} \wedge \bar{w}^{l} \wedge i_{\bar{w}_{j}}-2 \delta_{j l} w^{k}-w^{j} \wedge w^{k} \wedge i_{w_{l}}\right] .
$$

From (2.13), we get

$$
\begin{aligned}
{\left[i_{\bar{w}_{i}}, \mathcal{T}\right]=\sqrt{-1} \sum_{j k l}(\partial \Theta)\left(w_{j}, w_{k}, \bar{w}_{l}\right) w^{k} \wedge } & {\left[i_{\bar{w}_{i}}, \bar{w}^{l}\right] \wedge i_{\bar{w}_{j}} } \\
& =\sqrt{-1} \sum_{j k}(\partial \Theta)\left(w_{j}, w_{k}, \bar{w}_{i}\right) w^{k} \wedge i_{\bar{w}_{j}} .
\end{aligned}
$$

By (2.12) and (2.14), the equation (2.11) implies that

$$
\partial \Theta=0 .
$$

Thus $\bar{\partial} \Theta=\overline{\partial \Theta}=0$ and $d \Theta=0$. This means that if $\Delta$ preserves the bigrading on $\Omega^{\bullet}(X)$, then $(X, J, \Theta)$ is Kähler.

Remark 2.3. After we sent our preliminary version to Professor Bryant, he sent us an easier proof which works also in the almost-complex case. Here is the argument:

Let $(X, J, \Theta)$ be an almost complex manifold with almost complex structure $J$ and $\Theta$ a real $(1,1)$-form as in (1.27). We suppose that the Hodge Laplacian $\Delta$ preserves the bigrading. In fact, we may only suppose that $\Delta$ sends $(0,1)$-forms to $(0,1)$-forms. In particular, $\Delta$ commutes with $J: T X \rightarrow T X$. Using the following lemma, this implies that $J$ is parallel with respect to the Levi-Civita connection $\nabla^{T X}$ on $\left(T X, g^{T X}\right)$. It is well-known (cf. [12]) that this condition is equivalent to the metric being Kähler.

Lemma 2.4. Let $\left(X, g^{T X}\right)$ be a Riemannian manifold and $L \in \mathscr{C}^{\infty}\left(X, \operatorname{End}\left(T^{*} X\right)\right)$. If $L$ commutes with the Hodge Laplacian $\Delta$ on 1 -forms, then $L$ is parallel with respect to the Levi-Civita connection $\nabla^{T X}$.

Proof. Let $\nabla^{\Lambda\left(T^{*} X\right)}$ (resp. $\left.\nabla^{\operatorname{End}\left(T^{*} X\right)}\right)$ be the connection on $\Lambda\left(T^{*} X\right)\left(\operatorname{resp} . \operatorname{End}\left(T^{*} X\right)\right)$ induced by the Levi-Civita connection $\nabla^{T X}$ on $\left(T X, g^{T X}\right)$.

For $\nabla^{F}$ a connection on a vector bundle $F$, let $\Delta^{F}$ be the Bochner Laplacian on $F$ associated to $\nabla^{F}$. By Definition, for $\left\{e_{j}\right\}_{j}$ an orthonormal frame of $\left(T X, g^{T X}\right)$, we have

$$
\Delta^{F}=-\sum_{j}\left[\left(\nabla_{e_{j}}^{F}\right)^{2}-\nabla_{\nabla_{e_{j}}^{T X} e_{j}}^{F}\right]
$$

As $\nabla^{\Lambda\left(T^{*} X\right)}$ preserves the $\mathbb{Z}$-grading on $\Lambda\left(T^{*} X\right)$, we know the Bochner Laplacian $\Delta^{\Lambda\left(T^{*} X\right)}$ on $\Lambda\left(T^{*} X\right)$ associated to $\nabla^{\Lambda\left(T^{*} X\right)}$, also preserves the $\mathbb{Z}$-grading on $\Omega(X)$. One can relate $\Delta$ and $\Delta^{\Lambda\left(T^{*} X\right)}$ by the Weitzenböck formula (cf. [1, §3.6]). In particular, if $\alpha \in \Omega^{1}(X)$, one has the equality

$$
\Delta \alpha=\Delta^{T^{*} X} \alpha+\operatorname{Ric} \alpha,
$$


where the Ricci curvature Ric is identified with a section of the bundle $\operatorname{End}\left(T^{*} X\right)$ by means of $g^{T X}$.

By (2.16) and (2.17), the principal symbol $\sigma_{2}(\Delta)$ of $\Delta$ is $\sigma_{2}(\Delta)(\xi)=|\xi|^{2} \operatorname{Id}_{\Lambda\left(T^{*} X\right)}$ for $\xi \in T^{*} X$. Thus $\sigma_{2}(\Delta L-L \Delta)=0$ and $\Delta L-L \Delta$ is a first order differential operator. We now compute the principal symbol $\sigma_{1}(\Delta L-L \Delta)$ by computing $\lim _{t \rightarrow \infty} t^{-1} e^{-i t f}(\Delta L-$ $L \Delta) e^{i t f}$ when $t \rightarrow+\infty$ for any $f \in \mathscr{C}^{\infty}(X)$. By (2.16) and (2.17), we know for any $s \in \mathscr{C}^{\infty}\left(X, T^{*} X\right)$

$$
\sigma_{1}(\Delta L-L \Delta)(d f) s=\lim _{t \rightarrow \infty} t^{-1} e^{-i t f}(\Delta L-L \Delta) e^{i t f} s=-2 i\left(\nabla_{e_{j}}^{\operatorname{End}\left(T^{*} X\right)} L\right) e_{j}(f) s .
$$

By assumption, one has

$$
\Delta L-L \Delta=0
$$

This implies $\sigma_{1}(\Delta L-L \Delta)=0$. Thus from (2.18), we know (2.19) implies

$$
\nabla^{\operatorname{End}\left(T^{*} X\right)} L=0
$$

The proof of Lemma 2.4 is completed.

Remark 2.5. In the first proof of Theorem 2.2, we use the generalized Kähler identity. When we began to clarify the situation of question 0.3 , when there is a distribution, we computed an analogue of the generalized Kähler identity in this case. This is the object of the following subsection, which is independant from Subsection 2.3.

2.2. A generalized sub-Kähler identity. The Chern connection $\nabla^{T_{h} X}$ on $T_{h} X$ induces a connection on $T X$ and on the bundle $\Lambda^{\bullet \bullet \bullet}\left(T^{*} X\right)([12, \S 1.2 .2])$. This connection is denoted by $\widetilde{\nabla}^{T X}$. In what follows, we identify $T_{h} X$ with $T^{(1,0)} X$ and thus we denote by $\nabla^{T^{(1,0)} X}$ the connection $\nabla^{T_{h} X}$ on $T^{(1,0)} X$. For $v \in \mathscr{C}^{\infty}\left(X, T^{(0,1)} X\right)$, we define $\nabla^{T^{(0,1)} X} v=\overline{\nabla^{T^{(1,0)} X} \bar{v}}$. Then $\widetilde{\nabla}^{T X}=\nabla^{T^{(1,0)} X} \oplus \nabla^{T^{(0,1)} X}$. Moreover, we denote by $T \in \Lambda^{2}\left(T^{*} X\right) \otimes T X$ the torsion of $\widetilde{\nabla}^{T X}$.

By identifying $N$ in (1.10) to $T_{h} X / W, N$ induces a holomorphic structure from $T_{h} X / W$. Let $\pi_{N}$ be the orthogonal projection from $T_{h} X$ onto $N$. We denote by $\langle$, the $\mathbb{C}$-bilinear form on $T X \otimes_{\mathbb{R}} \mathbb{C}$ induced by $g^{T X}$.

Let $h^{W}, h^{N}$ be the Hermitian metrics on $W, N$ induced by $h^{T_{h} X}$. Let $\nabla^{W}, \nabla^{N}$ be the Chern connections on $\left(W, h^{W}\right),\left(N, h^{N}\right)$. Then we have

$$
\nabla^{W}=\pi_{W} \nabla^{T_{h} X} \pi_{W}, \quad \nabla^{N}=\pi_{N} \nabla^{T_{h} X} \pi_{N} .
$$

As $W$ is a holomorphic subbundle, we know

$$
A=\nabla^{T_{h} X^{\prime \prime}}-\left(\nabla^{W^{\prime \prime}} \oplus \nabla^{N^{\prime \prime}}\right) \in T^{*(0,1)} X \otimes \operatorname{Hom}(N, W) .
$$

The adjoint $A^{*}$ of $A$ takes values in $T^{*(1,0)} X \otimes \operatorname{Hom}(W, N)$. Note that for $w \in W, v \in$ $N, U \in T X \otimes_{\mathbb{R}} \mathbb{C}$, we have

$$
\left\langle A^{*}(U) w, \bar{v}\right\rangle=\langle w, \overline{A(\bar{U})(v)}\rangle .
$$

Then, under the decomposition $T_{h} X=W \oplus N$, we have

$$
\nabla^{T_{h} X}=\left(\begin{array}{cc}
\nabla^{W} & A \\
-A^{*} & \nabla^{N}
\end{array}\right) .
$$


Let $\widetilde{\nabla}^{W}, \widetilde{\nabla}^{N}$ be the connection on $W_{\mathbb{R}}, N_{\mathbb{R}}$ induced by $\nabla^{W}, \nabla^{N}$ as above or as in [12, (1.2.35)]. Set

$$
{ }^{\oplus} \nabla^{T X}=\widetilde{\nabla}^{W} \oplus \widetilde{\nabla}^{N}
$$

Let $\widetilde{\nabla}^{W}, \oplus \widetilde{\nabla}^{T X}$ be the connections on $\Lambda\left(W_{\mathbb{R}}^{*}\right), \Lambda\left(T^{*} X\right)$ induced by $\nabla^{W},{ }^{\oplus} \nabla^{T X}$ as in [12, $\S 1.2 .2]$, respectively.

Let $\left\{w_{j}\right\}_{j=1}^{m}$ be an orthonormal frame of $T^{(1,0)} X$ such that $\left\{w_{j}\right\}_{j=1}^{n}$ is an orthonormal frame of $W$. Then by [12, Lemma 1.4.4], we have

$$
\partial=\sum_{j=1}^{m} w^{j} \wedge \widetilde{\nabla}_{w_{j}}^{T X}+\frac{1}{2} \sum_{j, k, l=1}^{m}\left\langle T\left(w_{j}, w_{k}\right), \bar{w}_{l}\right\rangle w^{j} \wedge w^{k} \wedge i_{w_{l}},
$$

and

$$
\begin{aligned}
\bar{\partial}^{*}= & -\sum_{j=1}^{m} i_{\bar{w}_{j}} \widetilde{\nabla}_{w_{j}}^{T X}-\sum_{j, k=1}^{m}\left\langle T\left(w_{j}, w_{k}\right), \bar{w}_{k}\right\rangle i_{\bar{w}_{j}} \\
& +\frac{1}{2} \sum_{j, k, l=1}^{m}\left\langle T\left(w_{j}, w_{k}\right), \bar{w}_{l}\right\rangle \bar{w}^{l} \wedge i_{\bar{w}_{k}} \wedge i_{\bar{w}_{j}} .
\end{aligned}
$$

Note that for any $1 \leq j, k \leq m, U \in T X$, we have

$$
\begin{aligned}
& \left(\widetilde{\nabla}_{U}^{T X} w^{k}, w_{j}\right)=-\left(w^{k}, \widetilde{\nabla}_{U}^{T X} w_{j}\right)=-\left\langle\bar{w}_{k}, \widetilde{\nabla}_{U}^{T X} w_{j}\right\rangle=\left\langle\widetilde{\nabla}_{U}^{T X} \bar{w}_{k}, w_{j}\right\rangle, \\
& \left(\widetilde{\nabla}_{U}^{T X} \bar{w}^{k}, \bar{w}_{j}\right)=\left\langle\widetilde{\nabla}_{U}^{T X} w_{k}, \bar{w}_{j}\right\rangle .
\end{aligned}
$$

From (2.24), for $1 \leq k \leq m, n+1 \leq \gamma \leq m$, we get

$$
\widetilde{\nabla}_{w_{k}}^{T X} \bar{w}_{\gamma}={ }^{\oplus} \nabla_{w_{k}}^{T X} \bar{w}_{\gamma}+\sum_{j=1}^{n}\left\langle\overline{A\left(\bar{w}_{k}\right) w_{\gamma}}, w_{j}\right\rangle \bar{w}_{j} .
$$

From (2.24), (2.28) and (2.29), we know that on $\Lambda^{\bullet \bullet \bullet}\left(T^{*} X\right)$,

$$
\begin{aligned}
\widetilde{\nabla}_{w_{k}}^{T X}= & { }^{\oplus} \widetilde{\nabla}_{w_{k}}^{T X} \\
& +\sum_{\beta=n+1}^{m} \sum_{j=1}^{n}\left[-\left\langle A^{*}\left(w_{k}\right) w_{j}, \bar{w}_{\beta}\right\rangle \bar{w}^{\beta} \wedge i_{\bar{w}_{j}}+\left\langle\overline{A\left(\bar{w}_{k}\right) w_{\beta}}, w_{j}\right\rangle w^{j} \wedge i_{w_{\beta}}\right] \\
= & { }^{\oplus} \widetilde{\nabla}_{w_{k}}^{T X}+\sum_{\beta=n+1}^{m} \sum_{j=1}^{n}\left\langle\overline{A\left(\bar{w}_{k}\right) w_{\beta}}, w_{j}\right\rangle\left(-\bar{w}^{\beta} \wedge i_{\bar{w}_{j}}+w^{j} \wedge i_{w_{\beta}}\right) .
\end{aligned}
$$

By (1.30), (2.25), (2.26) and (2.30), we know that

$$
\partial_{W}=\sum_{j=1}^{n} w^{j} \wedge\left(\widetilde{\nabla}_{w_{j}}^{W}+\frac{1}{2} \sum_{k, l=1}^{n}\left\langle T\left(w_{j}, w_{k}\right), \bar{w}_{l}\right\rangle w^{k} \wedge i_{w_{l}}\right) .
$$

From (2.30), we get for $n+1 \leq \alpha \leq m$,

$$
\pi_{W} i_{\bar{w}_{\alpha}} \widetilde{\nabla}_{w_{\alpha}}^{T X} \pi_{W}=-\sum_{j=1}^{n}\left\langle w_{j}, \overline{A\left(\bar{w}_{\alpha}\right) w_{\alpha}}\right\rangle i_{\bar{w}_{j}} .
$$


From (1.30), (2.27), (2.30) and (2.32), we get

$$
\begin{aligned}
\bar{\partial}_{W}^{*}= & \sum_{j=1}^{n}\left(-i_{\bar{w}_{j}} \widetilde{\nabla}_{w_{j}}^{W}-\sum_{k=1}^{m}\left\langle T\left(w_{j}, w_{k}\right), \bar{w}_{k}\right\rangle i_{\bar{w}_{j}}\right) \\
& +\frac{1}{2} \sum_{j, k, l=1}^{n}\left\langle T\left(w_{j}, w_{k}\right), \bar{w}_{l}\right\rangle \bar{w}^{l} \wedge i_{\bar{w}_{k}} \wedge i_{\bar{w}_{j}}+\sum_{\alpha=n+1}^{m} \sum_{j=1}^{n}\left\langle w_{j}, \overline{A\left(\bar{w}_{\alpha}\right) w_{\alpha}}\right\rangle i_{\bar{w}_{j}} \\
= & \sum_{j=1}^{n} i_{\bar{w}_{j}}\left\{-\widetilde{\nabla}_{w_{j}}^{W}+\frac{1}{2} \sum_{k, l=1}^{n}\left\langle T\left(w_{j}, w_{k}\right), \bar{w}_{l}\right\rangle i_{\bar{w}_{k}} \wedge \bar{w}^{l}\right. \\
& \left.+\sum_{\alpha=n+1}^{m}\left(\left\langle w_{j}, \overline{A\left(\bar{w}_{\alpha}\right) w_{\alpha}}\right\rangle-\left\langle T\left(w_{j}, w_{\alpha}\right), \bar{w}_{\alpha}\right\rangle\right)\right\} .
\end{aligned}
$$

We also generalize the definition of the operators $L=\Theta \wedge$ and $\Lambda$ its adjoint, by defining $\Theta_{W} \in \Lambda^{1,1}\left(W_{\mathbb{R}}^{*}\right)$ as the restriction to $\Lambda^{1,1}\left(W_{\mathbb{R}}^{*}\right)$ of $\Theta$. We thus get operators $L_{W}$ and $\Lambda_{W}$ on $\Lambda^{\bullet \bullet \bullet}\left(W_{\mathbb{R}}^{*}\right)$. By $(2.3)$, we have

$$
L_{W}=\sqrt{-1} \sum_{j=1}^{n} w^{j} \wedge \bar{w}^{j} \wedge, \quad \Lambda_{W}=-\sqrt{-1} \sum_{j=1}^{n} i_{\bar{w}_{j}} i_{w_{j}} .
$$

The Hermitian torsion operator is defined as in (2.4) and [12, (1.4.34)] by

$$
\mathcal{T}_{W}:=\left[\Lambda_{W}, \partial_{W} \Theta_{W}\right] .
$$

We have the analogue of [12, Theorem 1.4.11].

Proposition 2.6. Generalized sub-Kähler identity

$$
\begin{aligned}
{\left[\Lambda_{W}, \partial_{W}\right]=\sqrt{-1} } & \left(\bar{\partial}_{W}^{*}+\overline{\mathcal{T}}_{W}^{*}\right) \\
& -\sqrt{-1} \sum_{\alpha=n+1}^{m} \sum_{j=1}^{n}\left(\left\langle w_{j}, \overline{A\left(\bar{w}_{\alpha}\right) w_{\alpha}}\right\rangle-\left\langle T\left(w_{j}, w_{\alpha}\right), \bar{w}_{\alpha}\right\rangle\right) i_{\bar{w}_{j}} \pi_{W} .
\end{aligned}
$$

Proof. Set $\pi_{W, \perp}=\mathrm{Id}-\pi_{W}$. By (2.7), we have

$$
\pi_{W}[\Lambda, \partial] \pi_{W}=\sqrt{-1}\left(\bar{\partial}_{W}^{*}+\pi_{W} \overline{\mathcal{T}}^{*} \pi_{W}\right) .
$$

Note that

$$
\pi_{W} \Lambda \pi_{W}=\Lambda \pi_{W}
$$

From (2.38), we know

$$
\begin{aligned}
\pi_{W}[\Lambda, \partial] \pi_{W}=\pi_{W} \Lambda \pi_{W} \partial \pi_{W}+\pi_{W} \Lambda \pi_{W, \perp} \partial \pi_{W}-\pi_{W} \partial \pi_{W} \Lambda \pi_{W} & \\
= & {\left[\Lambda_{W}, \partial_{W}\right]+\pi_{W} \Lambda \pi_{W, \perp} \partial \pi_{W} }
\end{aligned}
$$

By (2.26) and (2.30), we have

$$
\begin{aligned}
\pi_{W} \Lambda \pi_{W, \perp} \partial \pi_{W}=-\sqrt{-1} \pi_{W} \sum_{\gamma=n+1}^{m} i_{\bar{w}_{\gamma}} i_{w_{\gamma}} \pi_{W, \perp} \partial \pi_{W} & \\
& =\sqrt{-1} \sum_{\alpha=n+1}^{m} \sum_{j=1}^{n}\left\langle w_{j}, \overline{A\left(\bar{w}_{\alpha}\right) w_{\alpha}}\right\rangle i_{\bar{w}_{j}} \pi_{W}
\end{aligned}
$$



have

$$
\pi_{W}[\Lambda, \partial \Theta] \pi_{W}=\left[\Lambda_{W}, \partial_{W} \Theta_{W}\right]+\pi_{W} \Lambda \pi_{W, \perp} \partial \Theta \pi_{W} .
$$

By [12, (1.2.48), (1.2.54)], we have

$$
\partial \Theta=\frac{\sqrt{-1}}{2} \sum_{i, j, k=1}^{m}\left\langle T\left(w_{i}, w_{j}\right), \bar{w}_{k}\right\rangle w^{i} \wedge w^{j} \wedge \bar{w}^{k} .
$$

From (2.3) and (2.42), we know

$$
\pi_{W} \Lambda \pi_{W, \perp} \partial \Theta \pi_{W}=-\sum_{\alpha=n+1}^{m} \sum_{j=1}^{n}\left\langle T\left(w_{\alpha}, w_{j}\right), \bar{w}_{\alpha}\right\rangle w^{j} \pi_{W} .
$$

Taking the adjoint of (2.41), from (2.35) and (2.43), we know

$$
\pi_{W} \mathcal{T}^{*} \pi_{W}=\mathcal{T}_{W}^{*}-\sum_{\alpha=n+1}^{m} \sum_{j=1}^{n}\left\langle T\left(\bar{w}_{\alpha}, \bar{w}_{j}\right), w_{\alpha}\right\rangle i_{w_{j}} \pi_{W}
$$

Thus

$$
\pi_{W} \overline{\mathcal{T}}^{*} \pi_{W}=\overline{\mathcal{T}}_{W}^{*}-\sum_{\alpha=n+1}^{m} \sum_{j=1}^{n}\left\langle T\left(w_{\alpha}, w_{j}\right), \bar{w}_{\alpha}\right\rangle i_{\bar{w}_{j}} \pi_{W} .
$$

Finally from (2.37), (2.39), (2.40) and (2.45), we get

$$
\begin{aligned}
{\left[\Lambda_{W}, \partial_{W}\right]=\sqrt{-1} } & \left(\bar{\partial}_{W}^{*}+\overline{\mathcal{T}}_{W}^{*}\right) \\
& -\sqrt{-1} \sum_{\alpha=n+1}^{m} \sum_{j=1}^{n}\left(\left\langle w_{j}, \overline{A\left(\bar{w}_{\alpha}\right) w_{\alpha}}\right\rangle+\left\langle T\left(w_{\alpha}, w_{j}\right), \bar{w}_{\alpha}\right\rangle\right) i_{\bar{w}_{j}} \pi_{W} .
\end{aligned}
$$

From (2.46), we get (2.36).

2.3. The proof of Theorem 2.1. Remember the construction of the holomorphic map $\varphi: F \widehat{\otimes} \Lambda^{\bullet} W^{*} \rightarrow \Lambda^{\bullet+2} W^{*}$ in Subsection [1.4. There we assumed for simplicity that this map has constant rank. For the purpose of Theorem 2.1, one can easily reduce to this case. Indeed, there exists an analytic subset $V$ of $X$ such that $\varphi: F \widehat{\otimes} \Lambda^{k} W^{*} \rightarrow \Lambda^{k+2} W^{*}$ has maximum rank on $X \backslash V$ for any $k$. In particular, $\varphi\left(F \widehat{\otimes} \Lambda^{k} W^{*}\right)$ forms a vector subbundle of $\Lambda^{k+2} W^{*}$ on $X \backslash V$ for any $k$. We can define the vector bundles $F_{\varphi}$ and $F_{\varphi, \perp}$ on $X \backslash V$ as before. On $X \backslash V$, we have the decompositions (1.32) and (1.33) for forms with compact support; in particular

$$
\mathcal{Q} \cap \Omega_{c}^{\bullet}(X \backslash V)=\mathscr{C}_{c}^{\infty}\left(X \backslash V, F_{\varphi, \perp} \widehat{\otimes} \bar{F}_{\varphi, \perp}\right) .
$$

As $X \backslash V$ is an open connected dense subset of $X$, the characteristic Laplacian $\Delta_{\mathcal{Q}}$ preserves the bigrading on $\mathcal{Q}$ if and only if it preserves the bigrading on $\mathcal{Q} \cap \Omega_{c}^{\bullet}(X \backslash V)$. Thus we can work on $X \backslash V$ instead of $X$.

From the above discussion, in the rest, we will assume that $\varphi\left(F \widehat{\otimes} \Lambda^{k} W^{*}\right)$ forms a vector subbundle of $\Lambda^{k+2} W^{*}$ on $X$ for any $k$. Then we can use the formalism developed in Subsection 1.4. 
By (1.1), (1.28), as in (2.5), we have

$$
\Delta_{\mathcal{Q}}=\left[\partial_{\mathcal{Q}}+\bar{\partial}_{\mathcal{Q}}, \partial_{\mathcal{Q}}^{*}+\bar{\partial}_{\mathcal{Q}}^{*}\right]=\square_{\mathcal{Q}}+\bar{\square}_{\mathcal{Q}}+\left[\partial_{\mathcal{Q}}, \bar{\partial}_{\mathcal{Q}}^{*}\right]+\left[\bar{\partial}_{\mathcal{Q}}, \partial_{\mathcal{Q}}^{*}\right]
$$

As $\square_{\mathcal{Q}}, \bar{\square}_{\mathcal{Q}}$ preserve the bigrading on $\mathcal{Q}$, and $\left[\partial_{\mathcal{Q}}, \bar{\partial}_{\mathcal{Q}}^{*}\right]: \mathcal{Q}^{\bullet, \bullet} \rightarrow \mathcal{Q}^{\bullet+1, \bullet-1}$, we know that $\Delta_{\mathcal{Q}}$ preserves the bigrading on $\mathcal{Q}$ if and only if

$$
\left[\partial_{\mathcal{Q}}, \bar{\partial}_{\mathcal{Q}}^{*}\right]=0
$$

We would like to understand the operator $\left[\partial_{\mathcal{Q}}, \bar{\partial}_{\mathcal{Q}}^{*}\right]$.

For $f \in \mathscr{C}^{\infty}(X)$, by (1.30), we have

$$
\partial_{W} f=\sum_{j=1}^{n} w_{j}(f) w^{j} \in W^{*},
$$

where $\left\{w_{j}\right\}_{j=1}^{m}$ is an orthonormal frame of $T^{(1,0)} X$ such that $\left\{w_{j}\right\}_{j=1}^{n}$ is an orthonormal frame of $W$. For $\xi \in T_{\mathbb{R}}^{*} X$, let $\xi^{*} \in T_{\mathbb{R}} X$ be the metric dual of $\xi$. In particular, if $\xi \in W^{*}$, then $\xi^{*} \in \bar{W}$.

Since $\mathcal{J}$ is stable by $d, \partial, \bar{\partial}$, as in (1.2), we have as maps on $\Omega(X)$,

$$
\begin{aligned}
& \pi_{\mathcal{Q}} \circ \partial \circ \pi_{\mathcal{Q}}=\pi_{\mathcal{Q}} \circ \partial, \\
& \pi_{\mathcal{Q}} \circ \bar{\partial} \circ \pi_{\mathcal{Q}}=\pi_{\mathcal{Q}} \circ \bar{\partial}, \quad \pi_{\mathcal{Q}} \circ \bar{\partial}^{*} \circ \pi_{\mathcal{Q}}=\bar{\partial}^{*} \circ \pi_{\mathcal{Q}} .
\end{aligned}
$$

Let $h^{F_{\varphi}}, h^{F_{\varphi, \perp}}$ be the Hermitian metrics on $F_{\varphi}, F_{\varphi, \perp}$ induced by $h^{\Lambda W^{*}}$ on $\Lambda W^{*}$, which is induced by $h^{W}$. We recall that $F_{\varphi}$ is a holomorphic vector subbundle of $\Lambda W^{*}$. As in (2.21), let $\nabla^{F_{\varphi}}, \nabla^{F_{\varphi, \perp}}$ be the Chern connections on $\left(F_{\varphi}, h^{F_{\varphi}}\right),\left(F_{\varphi, \perp}, h^{F_{\varphi, \perp}}\right)$. Let $\nabla^{\Lambda W^{*}}$ be the connection on $\Lambda W^{*}$ induced by $\nabla^{W}$, then $\nabla^{\Lambda W^{*}}$ is the Chern connection on $\left(\Lambda W^{*}, h^{\Lambda W^{*}}\right)$. Set

$$
B=\nabla^{\Lambda W^{* \prime \prime}}-\left(\nabla^{F_{\varphi}^{\prime \prime}} \oplus \nabla^{F_{\varphi, \perp}}\right) \in T^{*(0,1)} X \otimes \operatorname{Hom}\left(F_{\varphi, \perp}, F_{\varphi}\right) .
$$

The adjoint $B^{*}$ of $B$ takes values in $T^{*(1,0)} X \otimes \operatorname{Hom}\left(F_{\varphi}, F_{\varphi, \perp}\right)$. Then under the decomposition $\Lambda W^{*}=F_{\varphi} \oplus F_{\varphi, \perp}$, we have

$$
\nabla^{\Lambda W^{*}}=\left(\begin{array}{cc}
\nabla^{F_{\varphi}} & B \\
-B^{*} & \nabla^{F_{\varphi, \perp}}
\end{array}\right)
$$

We denote also $\pi_{\mathcal{Q}}$ the orthogonal projection from $\Lambda W^{*} \widehat{\otimes} \Lambda \bar{W}^{*}$ onto $F_{\varphi, \perp} \widehat{\otimes} \bar{F}_{\varphi, \perp}$, and $\pi_{\mathcal{Q}}^{\perp}=\operatorname{Id}_{\Lambda W^{*} \widehat{\otimes} \Lambda \bar{W}^{*}}-\pi_{\mathcal{Q}}$. Then

$$
\begin{aligned}
& \pi_{\mathcal{Q}}=\pi_{F_{\varphi, \perp}} \otimes \bar{\pi}_{F_{\varphi, \perp}}, \\
& \pi_{\mathcal{Q}}^{\perp}=\pi_{F_{\varphi}} \otimes \bar{\pi}_{F_{\varphi, \perp}}+\pi_{F_{\varphi, \perp}} \otimes \bar{\pi}_{F_{\varphi}}+\pi_{F_{\varphi}} \otimes \bar{\pi}_{F_{\varphi}} .
\end{aligned}
$$

Lemma 2.7. The operator $\left[\partial_{\mathcal{Q}}, \bar{\partial}_{\mathcal{Q}}^{*}\right]$ is a first order differential operator acting on $F_{\varphi, \perp} \widehat{\otimes} \bar{F}_{\varphi, \perp}$. Its principal symbol, evaluated on $\xi \in T^{*} X$, is $\sqrt{-1}$ times

$$
\begin{aligned}
\pi_{\mathcal{Q}} \sum_{j, k=1}^{n} i_{\bar{w}_{j}} w^{k} \wedge\left[\left(\xi_{W}, T\left(w_{j}, w_{k}\right)\right)-\left(\xi, \pi_{N}\left[w_{j}, w_{k}\right]\right)\right] \pi_{\mathcal{Q}} & \\
& +\sum_{j=1}^{n}\left(B^{*}\left(w_{j}\right) \pi_{F_{\varphi}} \xi_{W}\right) \widehat{\otimes} i_{\bar{w}_{j}}-\pi_{\mathcal{Q}} \sum_{j=1}^{n} w^{j} \widehat{\otimes} i_{\left(\xi_{W}\right)^{*}} \overline{B\left(\overline{w_{j}}\right)}
\end{aligned}
$$

where $\xi_{W}$ is the orthogonal projection of $\xi$ on $W^{*}$ 
Remark 2.8. Theorem 2.1 is an easy corollary of (2.55). Indeed, if we take $\xi$ a holomorphic one-form which is orthogonal to $W^{*}$, the principal symbol is

$$
-\sqrt{-1} \pi_{\mathcal{Q}} \sum_{j, k=1}^{n} i_{\bar{w}_{j}} w^{k} \wedge\left(\xi, \pi_{N}\left[w_{j}, w_{k}\right]\right) \pi_{\mathcal{Q}}
$$

Evaluating at $\bar{w}^{j}$, which belongs to $F_{\varphi, \perp}$, one gets

$$
\sqrt{-1} \sum_{k=1}^{n}\left(\xi, \pi_{N}\left[w_{j}, w_{k}\right]\right) w^{k}
$$

This term vanishes for any $j$ and $\xi$ if and only if the distribution is involutive, which shows Theorem 2.1.

Proof of Lemma 2.7. Note that for $\xi \in W^{*}, \psi \in F_{\varphi, \perp} \widehat{\otimes} \bar{F}_{\varphi, \perp}$, by (1.16), (1.24) and (2.54), we know that $\pi_{\mathcal{Q}}^{\perp}(\xi \wedge \psi) \in F_{\varphi} \widehat{\otimes} \bar{F}_{\varphi, \perp}$. Thus by (1.24) $), i_{\xi^{*}} \pi_{\mathcal{Q}}^{\perp}(\xi \wedge \psi) \in F_{\varphi} \widehat{\otimes} \bar{F}_{\varphi, \perp}$, as $\xi^{*} \in \bar{W}$, and this implies

$$
\pi_{\mathcal{Q}} i_{\xi^{*}} \pi_{\mathcal{Q}}^{\perp} \xi \wedge \pi_{\mathcal{Q}}=0 \quad \text { for any } \xi \in W^{*}
$$

We compute now the principal symbol of $\left[\partial_{\mathcal{Q}}, \bar{\partial}_{\mathcal{Q}}^{*}\right]$ by computing the asymptotics of $e^{-i t f}\left[\partial_{\mathcal{Q}}, \bar{\partial}_{\mathcal{Q}}^{*}\right] e^{i t f}$ when $t \rightarrow+\infty$ for $f \in \mathscr{C}^{\infty}(X)$. By (1.31), (2.31) and (2.33), we have first

$$
\begin{gathered}
e^{-i t f} \partial_{\mathcal{Q}} e^{i t f}=i t \pi_{\mathcal{Q}} \partial_{W} f \wedge \pi_{\mathcal{Q}}+\partial_{\mathcal{Q}}, \\
e^{-i t f} \bar{\partial}_{\mathcal{Q}}^{*} e^{i t f}=-i t \pi_{\mathcal{Q}} i_{\left(\partial_{W} f\right)^{*}} \pi_{\mathcal{Q}}+\bar{\partial}_{\mathcal{Q}}^{*}
\end{gathered}
$$

Thus from (1.24) (2.57), the principal symbol of $\left[\partial_{\mathcal{Q}}, \bar{\partial}_{\mathcal{Q}}^{*}\right]$ as a second order differential operator is $\lim _{t \rightarrow \infty} t^{-2} e^{-i t f}\left[\partial_{\mathcal{Q}}, \bar{\partial}_{\mathcal{Q}}^{*}\right] e^{i t f}$, that is

$$
\begin{aligned}
& {\left[i \pi_{\mathcal{Q}} \partial_{W} f \wedge \pi_{\mathcal{Q}},-i \pi_{\mathcal{Q}} i_{\left(\partial_{W} f\right)^{*}} \pi_{\mathcal{Q}}\right]} \\
& \quad=\pi_{\mathcal{Q}} \partial_{W} f \wedge i_{\left(\partial_{W} f\right)^{*}} \pi_{\mathcal{Q}}+\pi_{\mathcal{Q}} i_{\left(\partial_{W} f\right)^{*}} \pi_{\mathcal{Q}} \partial_{W} f \wedge \pi_{\mathcal{Q}} \\
& \quad=-\pi_{\mathcal{Q}} i_{\left(\partial_{W} f\right)^{*}} \pi_{\mathcal{Q}}^{\perp} \partial_{W} f \wedge \pi_{\mathcal{Q}}=0
\end{aligned}
$$

here we use (2.56) in the last equality. The equation (2.58) means that $\left[\partial_{\mathcal{Q}}, \bar{\partial}_{\mathcal{Q}}^{*}\right]$ is a first order differential operator.

By (2.57), the principal symbol of $\left[\partial_{\mathcal{Q}}, \bar{\partial}_{\mathcal{Q}}^{*}\right]$ as a first order differential operator is $\lim _{t \rightarrow \infty} t^{-1} e^{-i t f}\left[\partial_{\mathcal{Q}}, \bar{\partial}_{\mathcal{Q}}^{*}\right] e^{i t f}$, that is

$$
i\left[\pi_{\mathcal{Q}} \partial_{W} f \wedge \pi_{\mathcal{Q}}, \bar{\partial}_{\mathcal{Q}}^{*}\right]-i\left[\partial_{\mathcal{Q}}, \pi_{\mathcal{Q}} i_{\left(\partial_{W} f\right)^{*}} \pi_{\mathcal{Q}}\right] .
$$

By (1.24) and (1.31), we get

$$
\begin{aligned}
{\left[\pi_{\mathcal{Q}} \partial_{W} f \wedge \pi_{\mathcal{Q}}, \bar{\partial}_{\mathcal{Q}}^{*}\right]=\pi_{\mathcal{Q}} \partial_{W} f \wedge \bar{\partial}_{W}^{*} \pi_{\mathcal{Q}} } & +\pi_{\mathcal{Q}} \bar{\partial}_{W}^{*} \pi_{\mathcal{Q}} \partial_{W} f \wedge \pi_{\mathcal{Q}} \\
& =\pi_{\mathcal{Q}} \bar{\partial}_{W}^{*}\left(\partial_{W} f\right) \pi_{\mathcal{Q}}-\pi_{\mathcal{Q}} \bar{\partial}_{W}^{*} \pi_{\mathcal{Q}}^{\perp} \partial_{W} f \wedge \pi_{\mathcal{Q}}
\end{aligned}
$$


Again by (1.24) and (1.31), we get

$$
\begin{aligned}
&-\left[\partial_{\mathcal{Q}}, \pi_{\mathcal{Q}} i_{\left(\partial_{W} f\right)^{*}} \wedge \pi_{\mathcal{Q}}\right] \\
&=-\pi_{\mathcal{Q}} i_{\left(\partial_{W} f\right)^{*}} \pi_{\mathcal{Q}} \partial_{W} \pi_{\mathcal{Q}}-\pi_{\mathcal{Q}} \partial_{W} i_{\left(\partial_{W} f\right)^{*}} \pi_{\mathcal{Q}} \\
& \quad=-\pi_{\mathcal{Q}} \partial_{W}\left(i_{\left(\partial_{W} f\right)^{*}}\right) \wedge \pi_{\mathcal{Q}}+\pi_{\mathcal{Q}} i_{\left(\partial_{W} f\right)^{*}} \pi_{\mathcal{Q}}^{\perp} \partial_{W} \pi_{\mathcal{Q}}
\end{aligned}
$$

By (2.33) and (2.50), we get

$$
\begin{gathered}
\bar{\partial}_{W}^{*}\left(\partial_{W} f\right)=\left[\bar{\partial}_{W}^{*}, \partial_{W} f\right]=-\left[\sum_{j=1}^{n} i_{\bar{w}_{j}} \widetilde{\nabla}_{w_{j}}^{W}, \partial_{W} f\right] \\
=-\sum_{j, k=1}^{n} i_{\bar{w}_{j}}\left[w_{j}\left(w_{k}(f)\right) w^{k}+w_{k}(f) \widetilde{\nabla}_{w_{j}}^{W} w^{k}\right] \\
=-\sum_{j, k=1}^{n} i_{\bar{w}_{j}} w^{k}\left[w_{j}\left(w_{k}(f)\right)-\left(\partial_{W} f, \nabla_{w_{j}}^{W} w_{k}\right)\right] .
\end{gathered}
$$

By (2.31) and (2.50), we get

$$
\begin{aligned}
-\partial_{W}\left(i_{\left(\partial_{W} f\right)^{*}}\right)=-\left[\partial_{W}, i_{\left(\partial_{W} f\right)^{*}}\right]=-\left[\sum_{k=1}^{n} w^{k} \widetilde{\nabla}_{w_{k}}^{W}, i_{\left(\partial_{W} f\right)^{*}}\right] \\
=-\sum_{j, k=1}^{n} w^{k} \wedge\left[w_{k}\left(w_{j}(f)\right) i_{\bar{w}_{j}}+w_{j}(f) i_{\nabla_{w_{k}} \bar{w}_{j}}\right] \\
=\sum_{j, k=1}^{n} i_{\bar{w}_{j}} w^{k}\left[w_{k}\left(w_{j}(f)\right)-\left(\partial_{W} f, \nabla_{w_{k}}^{W} w_{j}\right)\right] .
\end{aligned}
$$

By (2.24), (2.62) and (2.63), we get

$$
\begin{aligned}
& \bar{\partial}_{W}^{*}\left(\partial_{W} f\right)-\partial_{W}\left(i_{\left(\partial_{W} f\right)^{*}}\right) \\
&=\sum_{j, k=1}^{n} i_{\bar{w}_{j}} w^{k}\left[\left(\partial f,-\left[w_{j}, w_{k}\right]\right)+\left(\partial_{W} f, \nabla_{w_{j}}^{W} w_{k}-\nabla_{w_{k}}^{W} w_{j}\right)\right] \\
&=\sum_{j, k=1}^{n} i_{\bar{w}_{j}} w^{k}\left[\left(\partial_{W} f, T\left(w_{j}, w_{k}\right)\right)-\left(\partial f, \pi_{N}\left[w_{j}, w_{k}\right]\right)\right] .
\end{aligned}
$$

From (2.59)-(2.64), we know that the principal symbol of the first order differential operator $\left[\partial_{\mathcal{Q}}, \bar{\partial}_{\mathcal{Q}}^{*}\right]$ is $\sqrt{-1}$ times

$$
\begin{gathered}
\pi_{\mathcal{Q}} \sum_{j, k=1}^{n} i_{\bar{w}_{j}} w^{k}\left[\left(\partial_{W} f, T\left(w_{j}, w_{k}\right)\right)-\left(\partial f, \pi_{N}\left[w_{j}, w_{k}\right]\right)\right] \pi_{\mathcal{Q}} \\
-\pi_{\mathcal{Q}} \bar{\partial}_{W}^{*} \pi_{\mathcal{Q}}^{\perp} \partial_{W} f \wedge \pi_{\mathcal{Q}}+\pi_{\mathcal{Q}} i_{\left(\partial_{W} f\right)^{*}} \pi_{\mathcal{Q}}^{\perp} \partial_{W} \pi_{\mathcal{Q}}
\end{gathered}
$$


Now by (2.54), $\pi_{\mathcal{Q}}^{\perp} \partial_{W} f \wedge \pi_{\mathcal{Q}} \subset F_{\varphi} \otimes \bar{F}_{\varphi, \perp}$. By (1.24), (2.33) and (2.53), we know that

$$
\begin{aligned}
\pi_{\mathcal{Q}} \bar{\partial}_{W}^{*} \pi_{\mathcal{Q}}^{\perp} \partial_{W} f \wedge \pi_{\mathcal{Q}}=\pi_{\mathcal{Q}} \sum_{j=1}^{n}\left(-i_{\bar{w}_{j}} \widetilde{\nabla}_{w_{j}}^{W}\right) \pi_{\mathcal{Q}}^{\perp} \partial_{W} f \wedge \pi_{\mathcal{Q}} \\
\quad=\pi_{\mathcal{Q}} \sum_{j=1}^{n}\left(i_{\bar{w}_{j}} B^{*}\left(w_{j}\right) \otimes 1\right) \pi_{\mathcal{Q}}^{\perp} \partial_{W} f \wedge \pi_{\mathcal{Q}}=-\sum_{j=1}^{n}\left(B^{*}\left(w_{j}\right) \pi_{F_{\varphi}} \partial_{W} f \wedge\right) \widehat{\otimes}_{\bar{w}_{j}} .
\end{aligned}
$$

Let $P$ be the orthogonal projection from $\Lambda^{\bullet \bullet \bullet}\left(W_{\mathbb{R}}^{*}\right)$ onto $F_{\varphi, \perp} \otimes \bar{F}_{\varphi}$. Note that $\pi_{\mathcal{Q}}^{\perp} \partial_{W} \pi_{\mathcal{Q}} \subset$ $F_{\varphi} \otimes \Lambda \bar{W}^{*} \oplus F_{\varphi, \perp} \otimes \bar{F}_{\varphi}$, as $i_{\left(\partial_{W} f\right)^{*}} F_{\varphi} \otimes \Lambda \bar{W}^{*} \subset F_{\varphi} \otimes \Lambda \bar{W}^{*}$, from (2.31), (2.53) and (2.54), we have also

$$
\begin{aligned}
\pi_{\mathcal{Q}} i_{\left(\partial_{W} f\right)^{*}} \pi_{\mathcal{Q}}^{\perp} \partial_{W} \pi_{\mathcal{Q}} & =\pi_{\mathcal{Q}} i_{\left(\partial_{W} f\right)^{*}} P \partial_{W} \pi_{\mathcal{Q}} \\
& =\pi_{\mathcal{Q}} i_{\left(\partial_{W} f\right)^{*}} P \sum_{j=1}^{n} w^{j} \wedge \widetilde{\nabla}_{w_{j}}^{W} \pi_{\mathcal{Q}}=-\pi_{\mathcal{Q}} \sum_{j=1}^{n} w^{j} \widehat{\otimes} i_{\left(\partial_{W} f\right)^{*}} \overline{B\left(\overline{w_{j}}\right)}
\end{aligned}
$$

The proof of Lemma 2.7 is completed.

\section{REFERENCES}

[1] N. Berline, E. Gezler, M. Vergne, Heat Kernels and Dirac Operators, Grundl. Math. Wiss. 298, Springer-Verlag, Berlin, 1992.

[2] R. Bryant, S. Chern, R. Gardner, H. Goldschmidt and P. Griffiths, Exterior Differential Systems, Springer, 1990.

[3] R. Bryant and P. Griffiths, Characteristic Cohomology of Differential Systems (I): General Theory, Journal of the American Mathematical Society, 8, (1995), no. 3, 507-596.

[4] R. Bryant and P. Griffiths, Characteristic Cohomology of Differential Systems(II): Conservation laws for a class of parabolic equations Duke Mathematical Journal, 78, (1995), no. 3, 531-676.

[5] J. Carlson, M. Green and P. Griffiths, Variations of Hodge Structure Considered as an Exterior Differential System: Old and New Results, SIGMA 5 (2009), 087.

[6] S. S. Chern, On a generalization of Kähler geometry. Algebraic geometry and topology. A symposium in honor of S. Lefschetz, pp. 103-121. Princeton University Press, Princeton, N. J., 1957.

[7] J.-P. Demailly, Sur l'identité de Bochner-Kodaira-Nakano en géométrie hermitienne, Séminaire d'analyse P. Lelong-P. Dolbeault-H. Skoda, années 1983/1984, Lecture Notes in Math., vol. 1198, Springer, Berlin, 1986, pp. 88-97.

[8] M. Green, P. Griffiths and M. Kerr, Mumford-Tate domains. Boll. Unione Mat. Ital. (9) 3 (2010), no. 2, 281-307.

[9] P. Griffiths and J. Harris, Principles of algebraic geometry, Wiley-Interscience [John Wiley \& Sons], New York, 1978, Pure and Applied Mathematics.

[10] P. Griffiths, Exterior Differential Systems and Variations of Hodge Structures, http://publications.ias.edu/sites/default/files/eds.pdf, 2009.

[11] L. Hörmander, Hypoelliptic second order differential equations, Acta Mathematica, 119, (1967), no. $1,147-171$.

[12] X. Ma and G. Marinescu, Holomorphic Morse inequalities and Bergman kernels, Progress in Mathematics, vol. 254, Birkhäuser Boston Inc., Boston, MA, 2007, 422 pp.

[13] R. Montgomery, A Tour of Subriemannian Geometries, Their Geodesics and Applications, American Mathematical Soc., 2002.

[14] M. Taylor, Hypoelliptic (and Non-Hypoelliptic) Hodge Theory, unpublished work, 2010.

Université Paris Diderot - Paris 7, UFR de Mathématiques, Case 7012, 75205 Paris Cedex 13, France

E-mail address: danielj@math.jussieu.fr, ma@math.jussieu.fr 